\title{
TOPOLOGICALLY MASSIVE YANG-MILLS THEORY AND LINK INVARIANTS
}

\author{
by \\ Tuna Yildirim \\ A thesis submitted in partial fulfillment of the \\ requirements for the Doctor of Philosophy \\ degree in Physics \\ in the Graduate College of \\ The University of Iowa
}

December 2014

Thesis Supervisor: Professor Vincent G. J. Rodgers 
Copyright by TUNA YILDIRIM 2014

All Rights Reserved 


\author{
Graduate College \\ The University of Iowa \\ Iowa City, Iowa
}

\title{
CERTIFICATE OF APPROVAL
}

PH.D. THESIS

This is to certify that the Ph.D. thesis of

\section{Tuna Yildirim}

has been approved by the Examining Committee for the thesis requirement for the Doctor of Philosophy degree in Physics at the December 2014 graduation.

Thesis committee:

Vincent G. J. Rodgers, Thesis Supervisor

Charles Frohman

Craig E. Pryor

Wayne N. Polyzou

Mary Hall Reno 
To my parents:

Pakize and Yadigar Yildirim 


\section{ACKNOWLEDGEMENTS}

The idea of studying Chern-Simons link invariants modified with the presence of a small Yang-Mills term was proposed by my PhD advisor Prof. Vincent Rodgers and Prof. Parameswaran Nair. I am truly grateful and honored to work with them. I cannot thank enough for the time and effort they spent to help me. Especially, I want to thank my advisor Prof. Rodgers for taking me under his guidance, providing a great working environment, being a great mentor and a friend.

I thank the members of my thesis committee: Profs. Charles Frohman, Craig Pryor, Wayne Polyzou and Mary Hall Reno for their time, help and support. Especially to Prof. Charles Frohman for his encouragement and his help on knot theory. I thank professors Yannick Meurice, Yasar Onel, Sujeev Wickramasekara, Craig Kletzing, Maxim Khodas for their support and encouragement. I am thankful to Prof. Brian C. Hall for his time, support and help on geometric quantization. I also would like to acknowledge helpful comments from Profs. Edward Witten, Chris Baesley and Dr. Tudor Dimofte.

I am grateful to the current and previous members of the Diffeomorphism and Geometry Research Group: Dr. Brad Button, Chris Doran, Delalcan Kilic, Dr. Xiaolong Liu, Dr. Leo Rodriguez, Dr. Kory Stiffler and Catherine Whiting for their support and true friendship. Leo Rodriguez helped me so much, he was almost like a co-advisor to me during my first years in Iowa. Brad Button's support and friendship helped me go through some of the most difficult stages in graduate school. I thank Marc Herrmann for very helpful discussions and his support. I also would like to thank Cuneyt Sahin for his endless support 
and being a true friend.

I would like to thank my students Suzanne Carter, Wade Bloomquist and Hart Goldman for their interest in this research and checking some of my calculations.

I would like to thank the Physics and Astronomy department staff Debbie Foreman, Heather Mineart, Jeanne Mullen, Chris Stevens, Ron Vogel and Aaron Votroubek for being so helpful throughout my graduate school years.

I would like to thank the Beyond Center for Fundamental Concepts in Science at Arizona State University for their hospitality, where I wrote the final sections of this thesis. Especially Profs. Maulik Parikh and Tanmay Vachaspati, Dr. Eray Sabancilar and Subir Sabharwal for their support and helpful comments.

Finally, I would like to thank my family. Without their love and support, it would be impossible for me to accomplish anything. I cannot thank my parents Pakize and Yadigar Yildirim enough for their support and sacrifices. I am grateful to my brother Bora Yildirim for his support and encouragement.

This work was partially supported by National Science Foundation grant NSF1067889. 


\begin{abstract}
In this thesis, topologically massive Yang-Mills theory is studied in the framework of geometric quantization. This theory has a mass gap that is proportional to the topological mass $m$. Thus, Yang-Mills contribution decays exponentially at very large distances compared to $1 / m$, leaving a pure Chern-Simons theory with level number $k$. The focus of this research is the near Chern-Simons limit of the theory, where the distance is large enough to give an almost topological theory, with a small contribution from the Yang-Mills term. It is shown that this almost topological theory consists of two copies of Chern-Simons with level number $k / 2$, very similar to the Chern-Simons splitting of topologically massive AdS gravity model. As $m$ approaches to infinity, the split parts add up to give the original Chern-Simons term with level $k$. Also, gauge invariance of the split Chern-Simons theories is discussed for odd values of $k$. Furthermore, a relation between the observables of topologically massive Yang-Mills theory and Chern-Simons theory is obtained. It is shown that one of the two split Chern-Simons pieces is associated with Wilson loops while the other with 't Hooft loops. This allows one to use skein relations to calculate topologically massive Yang-Mills theory observables in the near Chern-Simons limit. Finally, motivated with the topologically massive AdS gravity model, Chern-Simons splitting concept is extended to pure Yang-Mills theory at large distances. It is shown that pure Yang-Mills theory acts like two Chern-Simons theories with level numbers $k / 2$ and $-k / 2$ at large scales. At very large scales, these two terms cancel to make the theory trivial, as required by the existence of a mass gap.
\end{abstract}




\section{TABLE OF CONTENTS}

LIST OF FIGURES . . . . . . . . . . . . . . . . . . . . v viii

\section{CHAPTER}

$1 \quad$ INTRODUCTION . . . . . . . . . . . . . . . . . . . . . 1

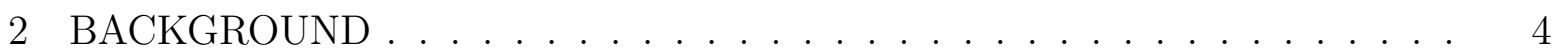

2.1 Yang-Mills Theory . . . . . . . . . . . . . . . . . 4

2.2 Problems of Quantum Chromodynamics . . . . . . . . . . . . . . 5

2.3 Why $2+1$ Dimensions? . . . . . . . . . . . . . . . . . 6

2.4 Chern-Simons Theory . . . . . . . . . . . . . . . . . 8

2.5 Topologically Massive Yang-Mills Theory . . . . . . . . . . . . . . . . . 9 9

2.6 The Classical Equivalence . . . . . . . . . . . . . . . . . . . . . . . 10

2.7 Topologically Massive AdS Gravity . . . . . . . . . . . . . . . . . . 11

2.8 The Wess-Zumino-Witten Model . . . . . . . . . . . . . . . . . . . . 13

2.9 Wilson Loops . . . . . . . . . . . . . . . . . . . . . 17

2.9.1 Confinement ...................... . . . 19

2.9 .2 't Hooft Loop Operator . . . . . . . . . . . . . . . 23

2.10 Knot Theory . . . . . . . . . . . . . . . . . . . . 23

2.10.1 History of Knot Theory . . . . . . . . . . . . . . . 23

2.10.2 The Jones Polynomial and Skein Relations . . . . . . . . . . . 24

2.10.3 An Example: Jones Polynomial of a Trefoil Knot . . . . . . . . . 25

2.10 .4 The HOMFLY Polynomial . . . . . . . . . . . . . 27

2.11 Chern-Simons Theory and Link Invariants . . . . . . . . . . . . . . . . 28

2.12 Geometric Quantization . . . . . . . . . . . . . . 33

2.12.1 Phase Space Geometry . . . . . . . . . . . . . . 34

2.12.2 Pre-quantization . . . . . . . . . . . . . . . . 35

2.12.3 Real Polarizations . . . . . . . . . . . . . . . . . 37

2.12.4 Half-Form Quantization . . . . . . . . . . . . . 39

2.12.5 Complex Polarizations . . . . . . . . . . . . . . . . 40

2.12.6 Geometric Quantization of Gauge Theories . . . . . . . . . . . 43

3 GEOMETRIC QUANTIZATION OF CHERN-SIMONS THEORY . . . . . . 46

3.1 Gauss' Law . . . . . . . . . . . . . . . . . . . . . . . . . . . . . . . . 48

3.2 The Wave-Functional . . . . . . . . . . . . . . . . . . . . 48

3.3 The Measure . . . . . . . . . . . . . . . . . . . 49

3.4 The $\Sigma=S^{1} \times S^{1}$ Case . . . . . . . . . . . . . . . . . 51

3.5 Wilson Loops in Chern-Simons Theory . . . . . . . . . . . . . . 51 
4 TOPOLOGICALLY MASSIVE YANG-MILLS THEORY . . . . . . . . 54

4.1 Gauss' Law . . . . . . . . . . . . . . . . . . . . . . . . 556

4.2 The Wave-Functional . . . . . . . . . . . . . . . . . . 56

4.2.1 The Schrödinger's Equation . . . . . . . . . . . . . 58

4.3 The Measure . . . . . . . . . . . . . . . . . . . . . . . 60

4.4 Wilson Loops in Topologically Massive Yang-Mills Theory . . . . . . . 663

5 2+1D PURE YANG-MILLS THEORY AT LARGE DISTANCES . . . . . . 6 66

5.1 Yang-Mills Theory In $2+1$ Dimensions . . . . . . . . . . . . . . 66 6

5.2 The Wave-Functional . . . . . . . . . . . . . . . . . . . . . . . 68

5.2 .1 The Schrödinger's Equation . . . . . . . . . . . . . . 70 70

5.3 The Measure . . . . . . . . . . . . . . . . . . . . . . . . . . . . . . 72

5.4 Chern-Simons Splitting . . . . . . . . . . . . . . . 73

6 CONCLUSION $\ldots \ldots \ldots \ldots \ldots \ldots$

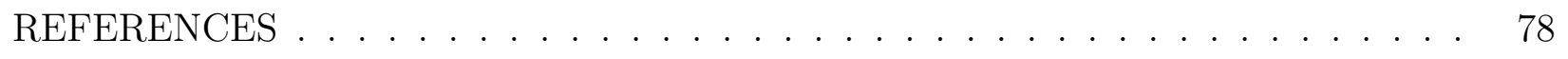




\section{LIST OF FIGURES}

Figure

2.1 Path $C \ldots \ldots \ldots \ldots \ldots \ldots \ldots$

2.2 A magnetic monopole-antimonopole pair placed in an electric superconductor. . 20

2.3 A rectangular loop for a quark-antiquark pair. . . . . . . . . . . 22

2.4 Skein Relations: Three different versions of a crossing on a link is depicted above. $V_{L_{+}}, V_{L_{-}}$and $V_{L_{0}}$ are the polynomials of the link with the corresponding version of the crossing. . . . . . . . . . . . . . . . . . 2 25

2.5 A skein relation to find the Jones polynomial of a link that consists of two separate

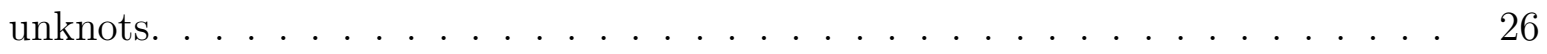

2.6 A skein relation to find the Jones polynomial of a Hopf link. . . . . . . . . 26

2.7 A skein relation to find the Jones polynomial of a right-handed trefoil knot. . . . 27

2.8 Knot diagrams for $\hat{L} \ldots \ldots \ldots \ldots \ldots$

2.9 Relating under-crossings with over-crossings. . . . . . . . . . . 31

3.1 Knot diagrams . . . . . . . . . . . . . . . . . . . . . . . . 52 


\section{CHAPTER 1 INTRODUCTION}

Chern-Simons theory has been extensively studied and is a very important part of mathematical physics, mostly because of its connection with the link invariants of knot theory. This was first demonstrated by Witten [1] using 2D conformal field theories related to Chern-Simons theory. Witten showed that Wilson loop expectation values of ChernSimons theory are given by link invariant polynomials which can be recursively calculated from skein relations. Later, Cotta-Ramusino et al. [2] derived skein relations for ChernSimons theory using only 3D field theory techniques. No matter what method is used, one crucial requirement for relating Wilson loop expectation values to link invariants is that the theory must be topological; in other words, the action must be metric free. Thus, it is not possible to do this with a Yang-Mills action because of its metric dependence. The main goal of this thesis is to find out how Chern-Simons link invariants are modified with the presence of a Yang-Mills term at large but finite distances, where the metric contribution is very small, hence the theory is almost topological.

It is well known that adding a Chern-Simons term to Yang-Mills action in $2+1$ dimensions gives mass to gauge bosons [3]. A considerable amount of work on topologically massive Yang-Mills theory was done by Deser, Jackiw and Templeton $[4-6]$ in the early 80s and some of the later work can be found in refs. 7f 17. Although topologically massive Yang-Mills theory is more complicated than Chern-Simons theory, there are surprising similarities between these theories, partly because conjugate momenta of both theories are given by gauge fields. An interesting example that shows the similarity of these two theories 
is the classical equivalence, first observed by Lemes et al [18, 19]. This equivalence shows that classically it is possible to write the topologically massive Yang-Mills action as a pure Chern-Simons action via a non-linear redefinition of the gauge fields. One would expect that it might be possible to extend this equivalence to quantum level, but we will show that phase space geometry does not allow this. Instead, we will obtain a more complicated equivalence between the observables of both theories at large but finite distances.

Since pure Yang-Mills theory in $2+1$ dimensions has a mass gap [20], the theory is trivial at very large distances. However, in topologically massive Yang-Mills theory that is not the case. The mass gap of this theory is proportional to the topological mass $m$ [15]. Studying large values of topological mass is equivalent to scaling up the metric or looking at large distances. In this thesis, we study the theory at large but finite distances by neglecting the second and higher order terms in $1 / m$, while keeping the first. This leads to an almost topological theory with a small contribution from Yang-Mills, which allows us to write topologically massive Yang-Mills theory observables in terms of Wilson loop expectation values of Chern-Simons theory. This means that, not only in the pure ChernSimons limit but also in the near Chern-Simons limit, one only needs skein relations to calculate topologically massive Yang-Mills theory observables. We also study pure YangMills theory at the same distance scale and obtain similar interesting results.

The thesis is organized as follows. Most of the necessary background knowledge is given in chapter 2, including introductions to knot theory (section 2.10) and geometric quantization section 2.12). In chapter 3, the methods described in chapter 2 are applied to Chern-Simons theory. In chapter 4 , similar methods are applied on a more complicated 
theory: topologically massive Yang-Mills. In chapter 5, we look at large distance behavior of pure Yang-Mils theory. Finally, in chapter 6 , the results obtained in chapter 4 and chapter 5 are discussed. 


\section{CHAPTER 2 \\ BACKGROUND}

\subsection{Yang-Mills Theory}

Yang-Mills Lagrangian is the non-abelian generalization of the Maxwell Lagrangian, written as

$$
\mathcal{L}=\frac{1}{2 e^{2}} \operatorname{Tr}\left(F_{\mu \nu} F^{\mu \nu}\right)
$$

where the constant $e^{2}$ is dimensionless in $4 \mathrm{D}$ and has the dimension of mass in $3 \mathrm{D}$. The curvature $F_{\mu \nu}$ is given by the commutator of the covariant derivatives, as $F_{\mu \nu}=\left[D_{\mu}, D_{\nu}\right]$, where $D_{\mu}=\partial_{\mu}+A_{\mu}$. $A_{\mu}$ can be expanded as $A_{\mu}=-i A_{\mu}^{a} t^{a}$, where $t^{a}$ are matrix representatives of the generators of Lie algebra $\left[t^{a}, t^{b}\right]=i f^{a b c} t^{c}$. Here, $t^{a}$ are hermitian matrices and $f^{a b c}$ are real valued structure constants. For $G=S U(N)$ where $G$ is the gauge group, $a, b, c=1,2, \ldots, N^{2}-1$. In the fundamental representation, the trace is normalized as $\operatorname{Tr}\left(t^{a} t^{b}\right)=\frac{1}{2} \delta^{a b}$. Expanding the trace, the Lagrangian 2.1 can be rewritten as

$$
\mathcal{L}=-\frac{1}{4 e^{2}} F_{\mu \nu}^{a} F^{a \mu \nu}
$$

Under a gauge transformation $A_{\mu} \rightarrow A_{\mu}^{g}=g A_{\mu} g^{-1}-\partial_{\mu} g g^{-1}, F_{\mu \nu}$ transforms covariantly, as

$$
F_{\mu \nu}(A) \rightarrow F_{\mu \nu}\left(A^{g}\right)=g F_{\mu \nu}(A) g^{-1}
$$

where $g \in G$. The cyclicity property of the trace makes the Lagrangian (2.1) gauge invariant. The equations of motion are given by

$$
D_{\mu} F^{\mu \nu}=0
$$


Covariant derivatives satisfy the Jacobi identity

$$
\left[D_{\mu},\left[D_{\nu}, D_{\alpha}\right]\right]+\left[D_{\nu},\left[D_{\alpha}, D_{\mu}\right]\right]+\left[D_{\alpha},\left[D_{\mu}, D_{\nu}\right]\right]=0,
$$

which leads to the Bianchi identity

$$
D_{\alpha} F_{\mu \nu}+D_{\mu} F_{\nu \alpha}+D_{\nu} F_{\alpha \mu}=0
$$

There is another important gauge invariant Lagrangian that can be constructed using $F_{\mu \nu}$. It is called the Pontryagin density and it is given by

$$
\mathcal{L}_{P}=-\frac{1}{32 \pi^{2}} \epsilon^{\mu \nu \alpha \beta} \operatorname{Tr}\left(F_{\mu \nu} F_{\alpha \beta}\right) .
$$

This Lagrangian can be written as a total derivative and it is related to the Chern-Simons Lagrangian as we will see in section 2.4.

Non-abelian Yang-Mills theory has been very successful in explaining weak and strong interactions. Thus, it is a very important part of the standard model.

\subsection{Problems of Quantum Chromodynamics}

The widely accepted model for Quantum Chromo-Dynamics(QCD) is an SU(3) gauge theory given by a Yang-Mills Lagrangian for gluons, plus a Dirac Lagrangian for quarks. An additional small topological term is also a possibility, to explain CP violation. This term is given by (2.7).

The correct QCD action is expected to have a few important features. One of these is called the "asymptotic freedom" which causes the quark bonds to become weak at high energies. Yang-Mills theory is shown to exhibit such behavior by Gross, Wilczek and Politzer, who received the Nobel Prize for their discovery in 2004. 
The second important feature is color confinement of quarks and gluons. Confinement causes the force between color charged particles to increase with distance. We will discuss this behavior in detail later, in section 2.9.

The third feature is having a "mass gap". Mass gap is the difference between the vacuum energy and the lowest energy state, which is simply the mass of the lightest particle in the theory. Gluons are massless but strong interactions force them to form massive composite particles called glueballs, leading to the existence of a mass gap in the theory. The lightest force carrier being massive, clearly limits the range of the theory. If the mass gap has the value $\Delta$, then the theory is trivial at very large distances compared to $1 / \Delta$.

In 3+1 dimensions, showing that the Yang-Mills action has these features turned out to be a very difficult task. This difficulty lead physicist to search for ways to simplify the problem.

\subsection{Why $2+1$ Dimensions?}

Studies in $2+1$ dimensional gauge theories mainly started in early 80s, after Feynman 21 pointed out that QCD in $3+1$ dimensions is too complicated to understand the qualitative behavior of the theory, since one needs to calculate 104 numbers at each point in space-time. To simplify the problem, he suggested a number of ways including decreasing the number of colors, decreasing the number of dimensions and studying just gluon systems without quarks. One or more of these methods can be used, depending on the qualitative feature one wants to understand. One of the most important of these qualitative features is confinement. Since even with no quarks there is a gluon confinement problem, studying 
just gluon systems is a nice and simpler way to study confinement. Also, switching from $3+1 \mathrm{D}$ to $2+1 \mathrm{D}$ significantly simplifies the problem. In $2+1 \mathrm{D}$ with the gauge group $\mathrm{SU}(3)$, in a no quark system, one needs to calculate only 24 numbers at each point in space-time compared to 104 in the full problem. $1+1 \mathrm{D}$ is exactly solvable but choosing it would be oversimplifying, because there are no propagating degrees of freedom. Although it is still mathematically interesting, this case would not be very helpful in studying the features of the theory that we are trying to understand. But $2+1 \mathrm{D}$ is non-trivial and has propagating degrees of freedom, which makes it more suitable for our interest.

An important progress in the $2+1 \mathrm{D}$ program came from two groups. Karabali, Kim and Nair 22 made an analytic prediction on the string tension(up to some approximation) and the other group, Teper, Lucini and Bringoltz [23 25] used lattice gauge theory Monte Carlo simulations to calculate it. Both groups had results that are in excellent agreement up to only $3 \%$ difference.

Three decades after Feynman's paper, a good understanding of 3+1 D Yang-Mills theory still seems out of reach. There have been important improvements in the $2+1$ program but the next step is not clear. Our goal is to study the $2+1$ D system with a Yang-MillsChern-Simons action to understand the transition of a topological theory to non-topological theory. Hopefully, this strategy will be helpful in filling a gap in the literature on $2+1 \mathrm{D}$ gauge theories. 


\subsection{Chern-Simons Theory}

Chern-Simons(CS) theory is a topological field theory given by the action

$$
S_{C S}=-\frac{k}{4 \pi} \int d^{3} x \epsilon^{\mu \nu \alpha} \operatorname{Tr}\left(A_{\mu} \partial_{\nu} A_{\alpha}+\frac{2}{3} A_{\mu} A_{\nu} A_{\alpha}\right)
$$

This action was first studied in ref. 26. Chern and Simons observed that the Pontryagin density can be written as a total derivative,

$$
\epsilon^{\mu \nu \alpha \beta} \operatorname{Tr}\left(F_{\mu \nu} F_{\alpha \beta}\right)=4 \partial_{\beta} \epsilon^{\mu \nu \alpha \beta} \operatorname{Tr}\left(A_{\mu} \partial_{\nu} A_{\alpha}+\frac{2}{3} A_{\mu} A_{\nu} A_{\alpha}\right)
$$

This boundary term was interesting in its own right and gave birth to the Chern-Simons Lagrangian. Later, it was introduced to the physics literature by Jackiw and Templeton [27].

Since the Lagrangian of this theory is metric free, the theory is topological. The equations of motion are given by

$$
\frac{\delta S_{C S}}{\delta A_{\mu}^{a}}=\frac{k}{8 \pi} \epsilon^{\mu \nu \alpha} F_{\nu \alpha}^{a}=0
$$

Chern-Simon action is classically not gauge invariant. Under $A_{\mu} \rightarrow A_{\mu}^{g}=g A_{\mu} g^{-1}-\partial_{\mu} g g^{-1}$, the Lagrangian transforms as

$$
\mathcal{L}_{C S} \rightarrow \mathcal{L}_{C S}+\frac{k}{4 \pi} \epsilon^{\mu \nu \alpha} \partial_{\mu} \operatorname{Tr}\left(\partial_{\nu} g g^{-1} A_{\alpha}\right)+\frac{k}{12 \pi} \epsilon^{\mu \nu \alpha} \operatorname{Tr}\left(g^{-1} \partial_{\mu} g g^{-1} \partial_{\nu} g g^{-1} \partial_{\alpha} g\right)
$$

The total derivative term can be made vanished by choosing suitable boundary conditions. The last term however, does not vanish. Up to a constant, the integral of this term is called the winding number $\omega(g)$, given by

$$
\omega(g)=\frac{1}{24 \pi^{2}} \int d^{3} x \epsilon^{\mu \nu \alpha} \operatorname{Tr}\left(g^{-1} \partial_{\mu} g g^{-1} \partial_{\nu} g g^{-1} \partial_{\alpha} g\right) .
$$


With suitable boundary conditions, $\omega(g)$ is an integer. Now, we can write

$$
S_{C S}(A) \rightarrow S_{C S}\left(A^{g}\right)=S_{C S}(A)+2 \pi k \omega(g)
$$

As we have stated before, Chern-Simons action is classically not gauge invariant but it can be made gauge invariant at the quantum level by constraining $k$ to be an integer. In that case, the weight of the path integral $e^{i S_{C S}}$ does not change, thus the theory becomes gauge invariant. The integer $k$ is usually referred to as the "level number" of Chern-Simons theory.

We will discuss geometric quantization of this theory in (3).

\subsection{Topologically Massive Yang-Mills Theory}

Topologically massive Yang-Mills(TMYM) theory is a mixture of Chern-Simons(CS) and Yang-Mills(YM) theories. The action is given by $S_{T M Y M}=S_{C S}+S_{Y M}$. As an introduction, we will only discuss the abelian case for now, following ref. 28. The non-abelian theory will be discussed in chapter 4. The abelian Lagrangian is given by

$$
\mathcal{L}_{T M Y M}=-\frac{1}{4 m} F_{\mu \nu} F^{\mu \nu}+\frac{1}{4} \epsilon^{\mu \nu \alpha} A_{\mu} F_{\nu \alpha} .
$$

Here $m$ is called the topological mass for the following reason. The equations of motion are given by

$$
\partial_{\mu} F^{\mu \nu}+\frac{m}{2} \epsilon^{\nu \alpha \beta} F_{\alpha \beta}=0
$$

Using $* F^{\mu}=\frac{1}{2} \epsilon^{\mu \nu \alpha} F_{\nu \alpha}$ and $F^{\mu \nu}=\epsilon^{\mu \nu \alpha} * F_{\alpha}$ we can rewrite the equations of motion as

$$
\epsilon^{\mu \nu \alpha} \partial_{\mu} * F_{\alpha}+m * F^{\nu}=0
$$

Multiplying both sides by the $\epsilon$ tensor gives,

$$
\partial_{\mu} * F_{\nu}-\partial_{\nu} * F_{\mu}=m F_{\mu \nu}
$$


Then taking the covariant derivative of both sides and using the Bianchi identity $\partial_{\mu} * F^{\mu}=0$, we get

$$
\left(\partial_{\mu} \partial^{\mu}+m^{2}\right) * F_{\nu}=0
$$

It can be seen from (2.18), the additional topological(Chern-Simons) term in the Lagrangian gives mass to $* F_{\nu}$, hence the name topological mass.

\subsection{The Classical Equivalence}

As shown by Lemes et al. [18], [19], classically it is possible to write the TMYM action as a pure CS action, via this nonlinear gauge field redefinition:

$$
\hat{A}_{\mu}=A_{\mu}+\sum_{n=1}^{\infty} \frac{1}{m^{n}} \theta_{\mu}^{n}(D, F)
$$

and the first three $\theta_{\mu}^{n}$ coefficients are

$$
\begin{aligned}
\theta_{\mu}^{1} & =\frac{1}{4} \epsilon_{\mu \sigma \tau} F^{\sigma \tau} \\
\theta_{\mu}^{2} & =\frac{1}{8} D^{\sigma} F_{\sigma \mu} \text { and } \\
\theta_{\mu}^{3} & =-\frac{1}{16} \epsilon_{\mu \sigma \tau} D^{\sigma} D_{\rho} F^{\rho \tau}+\frac{1}{48} \epsilon_{\mu \sigma \tau}\left[F^{\sigma \rho}, F_{\rho}^{\tau}\right] .
\end{aligned}
$$

With this redefinition, the equivalence is given by

$$
S_{C S}(\hat{A})=S_{C S}(A)+S_{Y M}(A)
$$

where

$$
S_{C S}(\hat{A})=\frac{1}{2} \int_{\Sigma \times\left[t_{i}, t_{f}\right]} d^{3} x \epsilon^{\mu \nu \alpha} \operatorname{Tr}\left(\hat{A}_{\mu} \partial_{\nu} \hat{A}_{\alpha}+\frac{2}{3} \hat{A}_{\mu} \hat{A}_{\nu} \hat{A}_{\alpha}\right)
$$




$$
S_{C S}(A)=\frac{1}{2} \int_{\Sigma \times\left[t_{i}, t_{f}\right]} d^{3} x \epsilon^{\mu \nu \alpha} \operatorname{Tr}\left(A_{\mu} \partial_{\nu} A_{\alpha}+\frac{2}{3} A_{\mu} A_{\nu} A_{\alpha}\right)
$$

and

$$
S_{Y M}(A)=\frac{1}{4 m} \int_{\Sigma \times\left[t_{i}, t_{f}\right]} d^{3} x \operatorname{Tr} F_{\mu \nu} F^{\mu \nu}
$$

This equivalence is classical and cannot be exactly extended to the quantum level. Some work on addressing this issue was done by Quadri [29], using the BRST formulation.

Even though this equivalence does not work at the quantum level, it provides a good motivation to study the relation between TMYM theory and link invariants.

\subsection{Topologically Massive AdS Gravity}

In three dimensions, there is a gravitational analogue of TMYM theory, called the topologically massive gravity model. In this section, following refs. $30-32$, we will study this gravity model with a negative cosmological constant, which is called the topologically massive AdS gravity model. For a dynamical metric $\gamma_{\mu \nu}$, this model has the action

$$
S=\int d^{3} x\left[-\sqrt{-\gamma}(R-2 \Lambda)+\frac{1}{2 \mu} \epsilon^{\mu \nu \rho}\left(\Gamma_{\mu \beta}^{\alpha} \partial_{\nu} \Gamma_{\rho \alpha}^{\beta}+\frac{2}{3} \Gamma_{\mu \gamma}^{\alpha} \Gamma_{\nu \beta}^{\gamma} \Gamma_{\rho \alpha}^{\beta}\right)\right]
$$

This action can be written as two CS terms with defining

$$
A_{\mu b}^{ \pm}{ }_{\mu b}^{a}[e]=\omega_{\mu b}^{a}[e] \pm \epsilon_{b c}^{a} e_{\mu}^{c}
$$

where $e_{\mu}{ }^{a}$ is the dreibein and $\omega_{\mu}^{a} b[e]$ is the torsion-free spin connection. Then, the action 2.25 can be written as

$$
S[e]=-\frac{1}{2}\left(1-\frac{1}{\mu}\right) S_{C S}\left[A^{+}[e]\right]+\frac{1}{2}\left(1+\frac{1}{\mu}\right) S_{C S}\left[A^{-}[e]\right]
$$


where

$$
S_{C S}[A]=\frac{1}{2} \int \epsilon^{\mu \nu \rho}\left(A_{\mu b}^{a} \partial_{\nu} A_{\rho a}^{b}+\frac{2}{3} A_{\mu}^{a}{ }_{c}^{a} A_{\nu b}^{c} A_{\rho a}^{b}\right)
$$

For our interests, the main difference between this gravity model and TMYM theory is that the latter has a mass gap, therefore it has a topological behavior only at large distances. But the gravity model is topological irrespective of the value of $\mu$. In TMYM theory, large distances are obtained by taking large values of $m$, which corresponds to small values of $\mu$ in the gravitational analogue. On the other hand, the $\mu \rightarrow \infty$ limit of the gravity model is analogous to pure YM theory.

Now, let us focus on two important limits of 2.27). For small values of $\mu, 2.27$ can be written as a sum of two half CS theories as

$$
S[e] \approx \frac{1}{2 \mu} S_{C S}\left[A^{+}[e]\right]+\frac{1}{2 \mu} S_{C S}\left[A^{-}[e]\right]
$$

In the $\mu \rightarrow \infty$ limit, it is equal to the difference between two half CS theories, as

$$
S[e]=\frac{1}{2} S_{C S}\left[A^{-}[e]\right]-\frac{1}{2} S_{C S}\left[A^{+}[e]\right]
$$

At large distances, it would be interesting to find out whether or not a similar CS+CS type splitting appears in TMYM theory and a CS-CS type splitting in pure YM theory. These cases will be discussed in chapter 4 and chapter 5. But we will not study pure YM theory as the small $m$ limit of TMYM theory, since this would force us to focus on short distances instead of large, where the scale dependence is strong and no topological behavior can be expected. Thus, we will study pure YM as a separate theory in chapter 5. 


\subsection{The Wess-Zumino-Witten Model}

Wess-Zumino-Witten(WZW) ${ }^{1}$ model defines a conformal field theory in two dimensions. The action consists of two terms: a two dimensional kinetic term and a three dimensional topological term, as

$$
S_{W Z W}[U]=\frac{1}{8 \pi} \int_{\partial \mathcal{M}} d^{2} x \sqrt{g} g^{a b} \operatorname{Tr}\left(\partial_{a} U \partial_{b} U^{-1}\right)+\Gamma[U]
$$

where

$$
\Gamma[U]=-\frac{i}{12 \pi} \int_{\mathcal{M}} d^{3} x \epsilon^{\mu \nu \alpha} \operatorname{Tr}\left(U^{-1} \partial_{\mu} U U^{-1} \partial_{\nu} U U^{-1} \partial_{\alpha} U\right)
$$

and $\mathcal{M}$ is a three dimensional space with a closed boundary $\partial \mathcal{M} . U(x)$ are invertible matrices that are not necessarily unitary. $\Gamma[U]$ is called the Wess-Zumino term. This term was added to the kinetic term by Witten, to bosonize non-abelian fields in two-dimensions [34. The Wess-Zumino term is metric free, hence topological and its value is always an integer.

In an Euclidean space, using complex coordinates $z=x_{1}-i x_{2}$ and $\bar{z}=x_{1}+i x_{2}$, we can rewrite the action as

$$
S_{W Z W}[U]=\frac{1}{2 \pi} \int_{\partial \mathcal{M}} \operatorname{Tr}\left(\partial_{z} U \partial_{\bar{z}} U^{-1}\right)+\Gamma[U]
$$

The equations of motion for this model are given by

$$
\partial_{z}\left(U^{-1} \partial_{\bar{z}} U\right)=0 \text { and } \partial_{\bar{z}}\left(\partial_{z} U U^{-1}\right)=0
$$

which defines two currents $J_{z}=-\frac{1}{\pi} \partial_{z} U U^{-1}$ and $J_{z}=\frac{1}{\pi} U^{-1} \partial_{\bar{z}} U$. These currents are associated with infinitesimal left and right transformations $U \rightarrow(1+f(z)) U$ and $U \rightarrow U(1+g(\bar{z}))$. The action 2.33 is invariant under these transformations.

\footnotetext{
${ }^{1}$ This section is a review, following ref. 33 .
} 
The Wess-Zumino term has the following useful property,

$$
\Gamma[U M]=\Gamma[U]+\Gamma[M]-\frac{i}{4 \pi} \int_{\partial \mathcal{M}} d^{2} x \epsilon^{a b} \operatorname{Tr}\left(U^{-1} \partial_{a} U M^{-1} \partial_{b} M\right) .
$$

Equation (2.35) leads to a very important identity called the Polyakov-Wiegmann(PW) 35,36 identity, given by

$$
S_{W Z W}[U M]=S_{W Z W}[U]+S_{W Z W}[M]+\frac{1}{\pi} \int_{\partial \mathcal{M}} \operatorname{Tr}\left(U^{-1} \partial_{z} U \partial_{\bar{z}} M M^{-1}\right)
$$

A chiral splitting can be seen here, since there is only the anti-holomorphic derivative of $U$ and only the holomorphic derivative of $M$.

The WZW action can be used to calculate two-dimensional fermion determinants. Massless fermion Lagrangian naturally splits into two chiral terms as,

$$
\mathcal{L}=\bar{\psi} D_{z} \psi+\bar{\chi} D_{\bar{z}} \chi
$$

where $\psi$ and $\chi$ are chiral components of a spinor and $D_{i}$ is the covariant derivative in the adjoint representation, given by $D_{i}=\partial_{i}+A_{i} \cdot \operatorname{det} D_{z}$ and $\operatorname{det} D_{\bar{z}}$ can be evaluated using the WZW action. We will start by parametrizing the components of a gauge field $A$ with a complex matrix $U$. A good starting point would be taking $U$ to be something similar to

$$
U(x, 0, C)=\mathcal{P} \exp \left(-\int_{C}^{x} A_{i} d x^{i}\right)
$$

where $\mathcal{P}$ is the path ordering operator. Then the gauge field can be written as $A_{i}=-\partial_{i} U U^{-1}$. However, this is not a good way to parametrize the gauge fields, since it depends on the path $C$. A small deformation at point $x_{0}$ on the path results in a change

$$
\delta U(x, 0, C)=\mathcal{P} \exp \left(-\int_{C}^{x} A_{i} d x^{i}\right) F_{i j} \Sigma^{i j} \mathcal{P} \exp \left(-\int_{C}^{x_{0}} A_{i} d x^{i}\right)
$$


where $F_{i j}=\partial_{i} A_{j}-\partial_{j} A_{i}+\left[A_{i}, A_{j}\right]$ and $\Sigma^{i j}$ is the area element. To make the matrix path independent, $F_{i j}$ must be made vanished. In Chern-Simons theory this condition is satisfied on shell but Yang-Mills theory has no such behavior. To make sure that the curvature vanishes irrespective of the Lagrangian, $U$ can be chosen as

$$
U(x, 0, C)=\mathcal{P} \exp \left(-\int_{C}^{x} \mathcal{A}_{z} d z+A_{\bar{z}} d \bar{z}\right),
$$

where $\mathcal{A}_{z}$ is defined by $\partial_{z} A_{\bar{z}}-\partial_{\bar{z}} \mathcal{A}_{z}+\left[\mathcal{A}_{z}, A_{\bar{z}}\right]=0$. This flatness condition forces $\delta U$ to be zero under small deformations of the path $C$. With this definition, the gauge fields can be parametrized as

$$
A_{\bar{z}}=-\partial_{\bar{z}} U U^{-1} \text { and } A_{z}=U^{\dagger-1} \partial_{z} U^{\dagger}
$$

This parametrization is sometimes referred to as "Karabali-Nair parametrization" 37] in the literature. Also, $\mathcal{A}_{i}$ can be written as

$$
\mathcal{A}_{\bar{z}}=U^{\dagger-1} \partial_{\bar{z}} U^{\dagger} \text { and } \mathcal{A}_{z}=-\partial_{z} U U^{-1}
$$

The flatness condition $\partial_{z} A_{\bar{z}}-\partial_{\bar{z}} \mathcal{A}_{z}+\left[\mathcal{A}_{z}, A_{\bar{z}}\right]=0$ can be rewritten as $\mathcal{A}_{z}=\left(D_{\bar{z}}^{-1}\right) \partial_{z} A_{\bar{z}}$, which implies that the covariant derivative must be invertible. This requirement introduces a restriction on the topology of the space. In simply connected spaces, $D_{\bar{z}}$ does not have zero modes. However, when the space is not simply connected, for example $\Sigma=S^{1} \times S^{1}$, parametrization (2.41) does not work. In this case the correct parametrization is

$$
A_{\bar{z}}=-\partial_{\bar{z}} U U^{-1}+U\left(\frac{i \pi a}{\operatorname{Im} \tau}\right) U^{-1}
$$

where $a$ is a constant gauge field and $\tau$ is the modular parameter of the torus. 
Matrices $U$ and $U^{\dagger}$ transform like fermions, as

$$
U \rightarrow g U \text { and } U^{\dagger} \rightarrow U^{\dagger} g^{-1}
$$

To write 2D fermion determinants in terms of the WZW action, let us define an action as $\mathcal{S} \equiv \log \operatorname{det} D_{\bar{z}}=\operatorname{Tr} \log D_{\bar{z}}$. After doing point-splitting regularization on $D_{\bar{z}}^{-1}[33]$, the variation of the action can be written as

$$
\delta \mathcal{S}=-\frac{1}{\pi} \int d^{2} x \operatorname{Tr}\left(\mathcal{A}_{z} \delta A_{\bar{z}}\right)_{A}
$$

where label $A$ indicates the adjoint representation. At this point we need to switch to the fundamental representation using $\operatorname{Tr}\left(t^{a} t^{b}\right)_{A}=2 c_{A} \operatorname{Tr}\left(t^{a} t^{b}\right)_{F}$, where $c_{A}$ is the quadratic Casimir in the adjoint representation given by $c_{A} \delta^{a d}=f^{a b c} f^{d b c}$. This allows us to write 2.45 as the variation of $S_{W Z W}(U)$ up to a constant, as

$$
\begin{aligned}
\delta \mathcal{S} & =-\frac{2 c_{A}}{\pi} \int d^{2} x \operatorname{Tr}\left(\mathcal{A}_{z} \delta A_{\bar{z}}\right)_{F} \\
& =2 c_{A} \delta S_{W Z W}(U) .
\end{aligned}
$$

Now we can write $\operatorname{det} D_{\bar{z}}$ in terms of $S_{W Z W}$ as

$$
\operatorname{det} D_{\bar{z}}=\operatorname{det}\left(\partial_{\bar{z}}\right) \exp \left(2 c_{A} S_{W Z W}(U)\right)
$$

and $\operatorname{det} D_{z}$ is the complex conjugate of $\operatorname{det} D_{\bar{z}}$, written as

$$
\operatorname{det} D_{z}=\operatorname{det}\left(\partial_{z}\right) \exp \left(2 c_{A} S_{W Z W}\left(U^{\dagger}\right)\right)
$$

These determinants are not gauge invariant. Under infinitesimal gauge transformations, the action $\mathcal{S}$ transforms as

$$
\delta_{\epsilon} \mathcal{S}=-\frac{1}{\pi} \int d^{2} x \operatorname{Tr}\left(\partial_{z} A_{\bar{z}} \delta g g^{-1}\right)=\frac{1}{2 \pi} \int d^{2} x \varepsilon^{a} \partial_{z} A_{\bar{z}}^{a}
$$


where $A_{\mu}=-i A_{\mu}^{a} t^{a}, \operatorname{Tr}\left(t^{a}, t^{b}\right)_{F}=\frac{1}{2} \delta^{a b}$ and $g \approx 1-i t^{a} \varepsilon^{a}$ is used $(\varepsilon<<1)$.

Using (2.47) and (2.48), the full Dirac determinant can be written as

$$
\operatorname{det}\left(D_{\bar{z}} D_{z}\right)=\operatorname{det}\left(\partial_{\bar{z}} \partial_{z}\right) \exp \left[2 c_{A}\left(S_{W Z W}(U)+S_{W Z W}\left(U^{\dagger}\right)\right)\right]
$$

which is still not gauge invariant. A local counter term can be added to the exponential to complete the PW identity. Adding a local counter term is allowed, since it is equivalent to choosing a different regularization. The complete PW identity is

$$
S_{W Z W}\left(U^{\dagger} U\right)=S_{W Z W}(U)+S_{W Z W}\left(U^{\dagger}\right)+\frac{1}{\pi} \int d^{2} x \operatorname{Tr}\left(\partial_{\bar{z}} U U^{-1} U^{\dagger-1} \partial_{z} U^{\dagger}\right)
$$

The last term is the local counter term that we have added, which can be written as $-\frac{1}{\pi} \int d^{2} x \operatorname{Tr}\left(A_{\bar{z}} A_{z}\right)$. Now we have everything we need to write the full Dirac determinant as a gauge invariant function. Since $\operatorname{det}\left(\partial_{\bar{z}} \partial_{z}\right)$ is just a constant factor [38], the full Dirac determinant can be written as

$$
\operatorname{det}\left(D_{\bar{z}} D_{z}\right)=\text { constant } \times \exp \left(2 c_{A} S_{W Z W}(H)\right)
$$

where $H=U^{\dagger} U$. It can be seen from (2.44) that $H$ is $S U(N)$ gauge invariant, so is the Dirac determinant (2.52).

\subsection{Wilson Loops}

One of the most important observables in gauge theory is the gauge invariant Wilson Loop, which is given by the holonomy of the gauge field around a space-time loop. This observable is non-local, like electromagnetic flux. Following refs. 33, 39, construction of this observable can be done as follows. We look at the parallel transport of matter fields. Let 
$\phi$ be a matter field. Then, analogous to Riemannian geometry, its parallel transport can be analyzed with the covariant derivative as

$$
D_{\mu} \phi=\left(\partial_{\mu}+A_{\mu}\right) \phi=0
$$

This equation can be solved by $\phi=U \phi_{0}$ where $\phi_{0}$ is a constant and $U$ is given by

$$
C^{\mu}\left(\partial_{\mu}+A_{\mu}\right) U=0
$$

where $C^{\mu}$ are components of a vector tangent to a curve $C$. The reason for introducing a curve here is the non-existence of a general well-defined solution to the equation $\left(\partial_{\mu}+A_{\mu}\right) U=0$. The equation 2.54 is solved by

$$
\begin{aligned}
U\left(y, x, C, A_{\mu}\right) & =\prod_{i}\left(1-A_{\mu}\left(x_{i}\right) d x_{i}^{\mu}\right) \\
& =\mathcal{P} \exp \left(-\int_{C}^{y} A_{\mu} d x^{\mu}\right),
\end{aligned}
$$

where $\mathcal{P}$ is the path ordering operator and definitions of $x_{i}$ and $d x_{i}$ can be seen clearly in Figure 2.1.

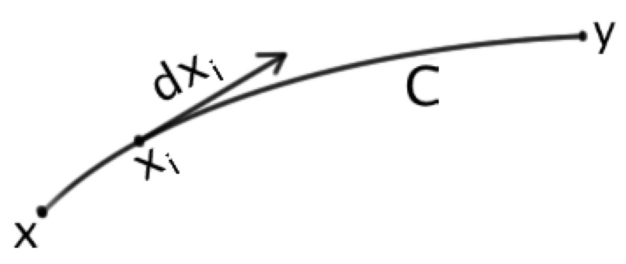

Figure 2.1: Path $C$

$\operatorname{Tr}\left(U\left(y, x, C, A_{\mu}\right)\right)$ is the definition of the Wilson line operator and it is neither gauge invari- 
ant nor covariant. Under $A \rightarrow A^{g}=g A g^{-1}-d g g^{-1}, U\left(y, x, C, A_{\mu}\right)$ transforms like

$$
U\left(y, x, C, A_{\mu}^{g}\right)=g(y) U\left(y, x, C, A_{\mu}\right) g(x)^{-1} .
$$

To build a gauge invariant quantity, instead of integrating over an open path, we can integrate over a closed loop, where $x$ and $y$ are the same point. Trace of $U\left(x, x, C, A_{\mu}\right)$ is gauge invariant and can be written as

$$
W_{R}(C)=\operatorname{Tr}_{R} \mathcal{P} \exp \left(-\oint_{C} A_{\mu} d x^{\mu}\right) .
$$

$W_{R}(C)$ is called the Wilson loop operator and subscript $R$ indicates the representation dependence of this observable.

\subsubsection{Confinement}

Wilson loop is an important tool to study confinement. If the theory is confined, the Wilson loop expectation value should satisfy the area law

$$
\left\langle W_{R}(C)\right\rangle=e^{-\sigma A_{C}}
$$

where $\sigma$ is a constant and $A_{C}$ is the area of the loop, given by the closed curve $C$. For a non-confining theory, the expectation value may follow the perimeter law

$$
\left\langle W_{R}(C)\right\rangle=e^{-m L_{C}}
$$

where $m$ is a constant and $L_{C}$ is the circumference of the loop. For a theory to have either 2.58 or 2.59 type of behavior, there has to be a mass gap since both laws follow a decay pattern at large scales.

To see how Wilson loop is related to confinement, following ref. 40, we will take a look at a quark-antiquark pair. The potential between a quark and an antiquark depends 
linearly on the separation $R$, except for very small values of $R$. For this case, the potential can be written as

$$
V_{q \bar{q}}(R) \stackrel{R \rightarrow \infty}{\longrightarrow} \sigma R
$$

$\sigma$ is a constant called the string tension. This behavior is explained by assuming a flux tube connecting the two quarks. The color analogue of electric field lines are confined in a tube-like region which acts like a string, connecting the quark-antiquark pair.

Confinement can be visualized with the help of an analogous electromagnetic system. Let us consider a hypothetical magnetic monopole-antimonopole pair that is placed inside an electric superconductor. In Figure 2.2 (a), the conductor is not in the super-conducting
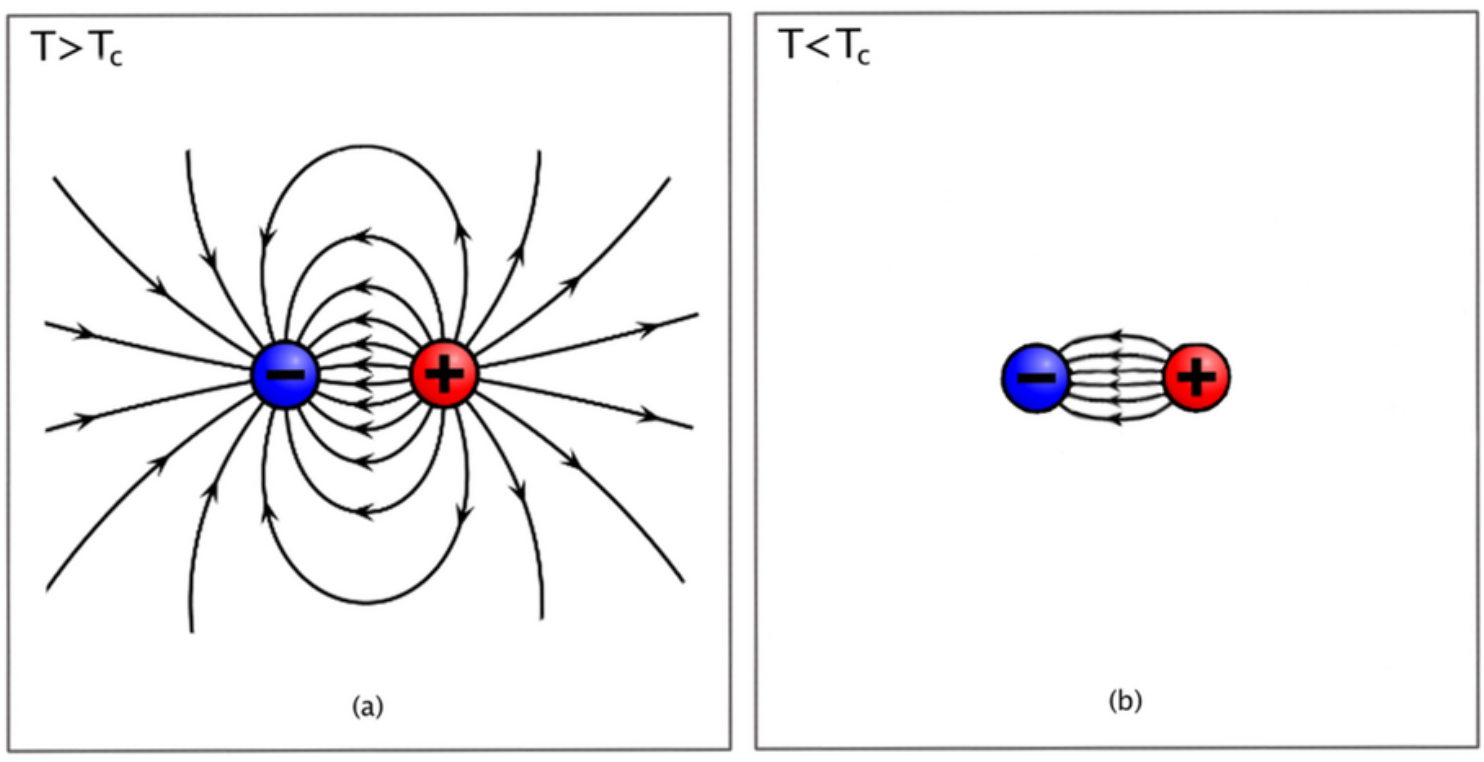

Figure 2.2: A magnetic monopole-antimonopole pair placed in an electric superconductor.

state, thus the magnetic field lines form the usual dipole pattern. Figure 2.2 (b) shows 
what happens to the field lines in the superconducting state. As a result of the Meissner effect, the magnetic field lines are compressed, forming a flux tube. What happens in QCD is analogous to this example, with interchanging the magnetic field lines with electric field lines and replacing the electric superconductor with a magnetic analogue. This explanation of confinement is known as the "dual super-conductor model" 441].

Now let us assume that the quark-antiquark pair $(q \bar{q})$ is heavy enough, so their kinetic energy can be neglected. In this case, the action is

$$
S=-\int_{0}^{T} d t V_{q \bar{q}}(R)=T V_{q \bar{q}}(R)
$$

This action is given by a quark-gluon interaction, thus it can be written as $S=-\int d^{4} x A_{\mu} j^{\mu}$. Since we assumed that the quark-antiquark pair is almost static, we can use the color charge density $\rho(x)=\delta^{3}(x)-\delta^{3}(x-R)$ and write

$$
j^{\mu}(x) A_{\mu}(x)=\rho(x) A_{0}(x)=\left(\delta^{3}(x)-\delta^{3}(x-R)\right) A_{0}(x) .
$$

Now, the action can be written as

$$
S=-\int d^{4} x A_{\mu} j^{\mu}=-\int_{0}^{T} d t\left(A_{0}(0, t)-A_{0}(R, t)\right)
$$

which should be equal to $T V_{q \bar{q}}(R)$, if the theory is confined. To relate this to a Wilson loop, we take the rectangular loop shown in Figure 2.3. 


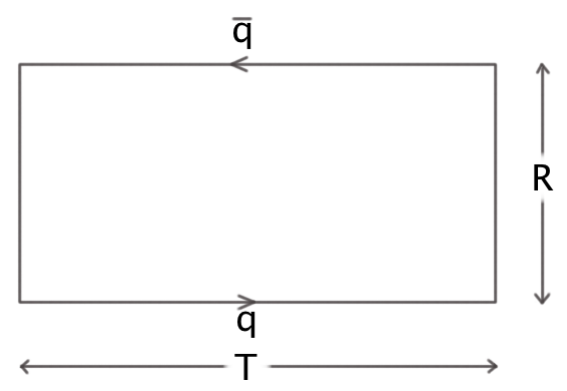

Figure 2.3: A rectangular loop

for a quark-antiquark pair.

If the measurement is taken for a long time, then $T \gg R$ can be used for approximation. With this approximation, the loop integral can be written as

$$
\begin{aligned}
-\oint A_{\mu} d x^{\mu}= & -\int_{0}^{T} d t A_{0}(0, t)+\int_{0}^{R} d x A_{x}(x, T) \\
& +\int_{T}^{0} d t A_{0}(R, t)+\int_{R}^{0} d x A(x, 0) \\
& { }^{\gtrsim} R^{R} T V(R) .
\end{aligned}
$$

With $V_{q \bar{q}}(R)=\sigma R$, the Wilson expectation value is given by

$$
\langle W(C)\rangle=\left\langle\operatorname{Tr} \mathcal{P} \exp \left(-\oint_{C} A_{\mu} d x^{\mu}\right)\right\rangle \approx e^{-\sigma R T} .
$$

Since $R T$ is the area of the loop, we obtain the area law $\left\langle W_{R}(C)\right\rangle \approx e^{-\sigma A_{C}}$ for $T \gg R$.

In some non-confining theories, Wilson loop expectation values may follow the perimeter law. This is not the case for topological theories, since they do not have a mass gap. They cannot satisfy a decay law due to their scale independence. For example, Chern-Simons theory Wilson loop expectation values are given by link invariant polynomials that depend only on the topology of a knot or link. Thus, the area or circumference of the loop is irrelevant. 
This will the subject of the section 2.10.

\subsection{2 't Hooft Loop Operator}

't Hooft loop operator is a dual version of the Wilson loop operator. It is defined in a similar way to Wilson loop with replacing the gauge field $A_{\mu}$ with its dual $\tilde{A}_{\mu}$, which is given by $E^{i}=\epsilon^{i j k} \partial_{j} \tilde{A}_{k}$. The 't Hooft loop operator is given by

$$
T(C)=\operatorname{Tr} \mathcal{P} \exp \left(-\oint_{C} \tilde{A}_{\mu} d x^{\mu}\right) .
$$

This operator, together with the Wilson loop in fundamental representation, satisfies the 't Hooft algebra,

$$
W_{F}(C) T\left(C^{\prime}\right)=e^{\frac{2 \pi i}{N} L\left(C, C^{\prime}\right)} T\left(C^{\prime}\right) W_{F}(C)
$$

\subsection{Knot Theory}

The mathematical definition of a knot is, a smooth embedding of a circle in a 3 or higher dimensional space. A finite union of non-intersecting knots is called a link. If a knot can be continuously deformed into another without crossing itself, these two knots are said to be equivalent.

\subsubsection{History of Knot Theory}

The history of knot theory dates back to 1867, when Lord Kelvin introduced his idea that atoms are knotted vortex tubes of ether [42]. P.G. Tait, a collaborator of Kelvin's, was the first to study the classification of knots and his discoveries has been called "Tait conjectures". An important improvement in knot theory was the discovery of the first link invariant polynomial in 1928: the "Alexander Polynomial" 43]. This invariant was not 
suitable enough to prove most of Tait conjectures. A more useful link invariant "Jones polynomial" was discovered in 1984 by Vaughan Jones [44], which made proving some of Tait conjectures possible 42$]$.

Knot theory was disregarded by the physics community for a long time after Kelvin's atom theory was proven wrong. But in late 1980s, Witten's work reintroduced knot theory to physics. Starting with Michael Atiyah's proposal [45], Witten showed that Chern-Simons theory Wilson loop expectation values can be described by the Jones polynomial or related invariants. This discovery opened a new path in understanding gauge theories and conformal field theories.

\subsubsection{The Jones Polynomial and Skein Relations}

The Jones polynomial of a link is a polynomial of a parameter $t$, denoted as $V_{L}(t)$. Jones polynomial of the mirror image of a knot is given by the transformation $t \rightarrow t^{-1}$; this is not the case for the Alexander polynomial since it cannot distinguish the knot from its mirror image.

The main tool to calculate the Jones polynomial is called a skein relation. To use a skein relation, one needs to take the projection of a three-dimensional link on a twodimensional surface. This leads to having crossings on the projection. Using a skein relation is basically doing surgery on one of these crossings. Jones polynomials satisfy the following skein relation,

$$
t^{-1} V_{L_{+}}+t V_{L_{-}}=\left(t^{1 / 2}-t^{-1 / 2}\right) V_{L_{0}}
$$

with the knot diagrams given in Figure 2.4. 

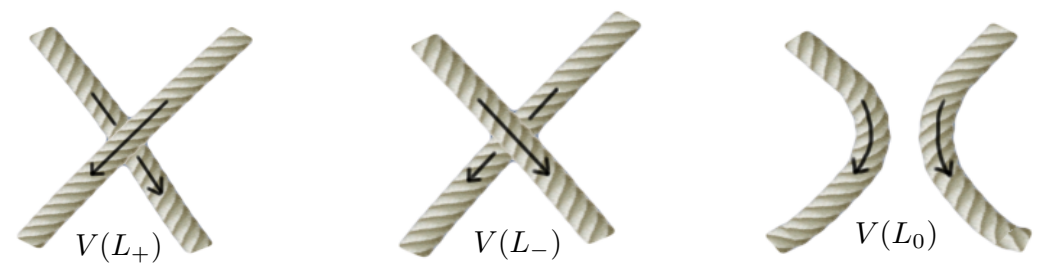

Figure 2.4: Skein Relations: Three different versions of a crossing on a link is depicted above. $V_{L_{+}}, V_{L_{-}}$and $V_{L_{0}}$ are the polynomials of the link with the corresponding version of the crossing.

The skein relation can be used recursively to calculate the polynomials of complicated links, starting with the normalization condition for the unknot(or simply, a circle) $V_{o}(t)=1$.

The Jones polynomial of a link that consists of an odd number of knots is always a Laurent polynomial of $\sqrt{t}$; for an even number, it is a polynomial of $t$.

\subsubsection{An Example: Jones Polynomial of a Trefoil Knot}

Now as an example, we will calculate the Jones polynomial for the right-handed trefoil knot. We start with two twisted unknots to get the polynomial of a link that consists of two separate unknots, as shown in Figure 2.5. In all upcoming figures of this section, the projection of the link represents the polynomial. Surgery will be done only on the crossing with arrows on it, which indicate the orientation of the knots. An orientation is necessary, since skein relations are meaningless without one. Without an orientation, the first two diagrams in Figure 2.4 would be topologically equivalent, since one of them is just the rotated version of the other one. 

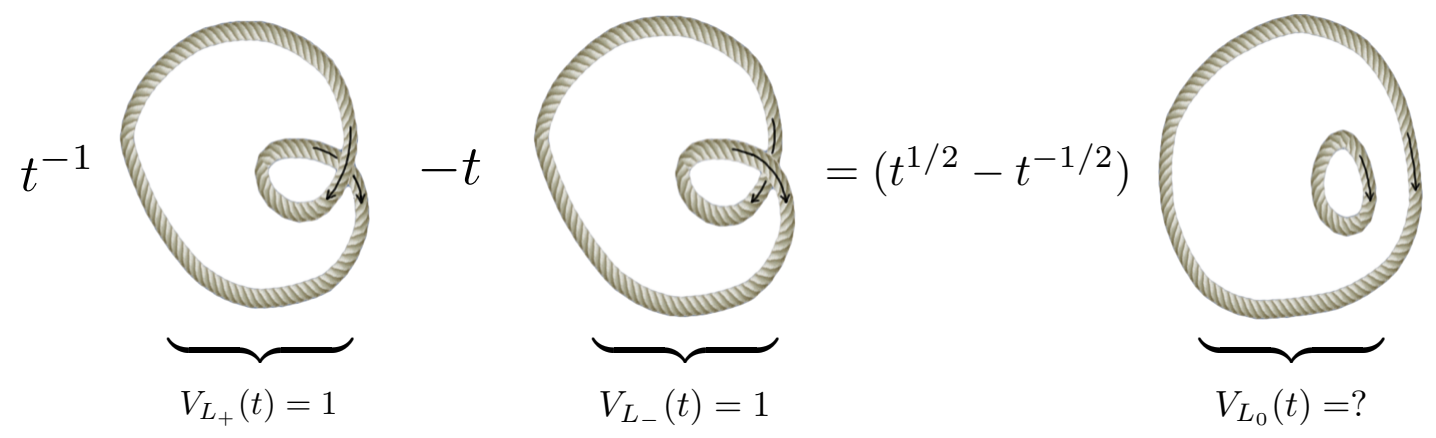

Figure 2.5: A skein relation to find the Jones polynomial of a link that consists of two separate unknots.

Solving the skein relation in Figure 2.5 for $V_{L_{0}}$, we get

$$
V_{L_{0}}(t)=-t^{1 / 2}-t^{-1 / 2}
$$

The next polynomial we need, is for the Hopf link, which consists of two unknots that go through each other as shown in the first term of Figure 2.6.

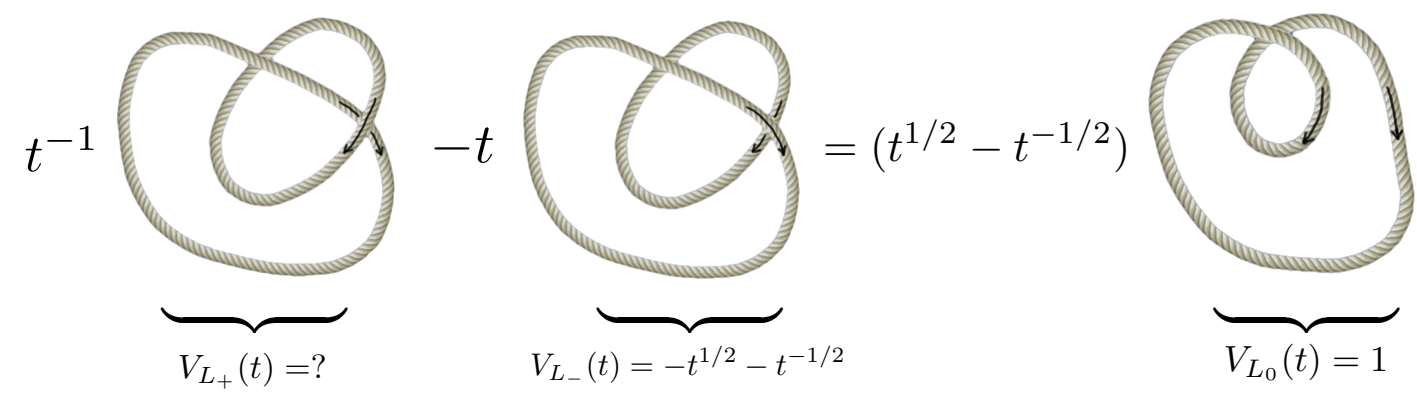

Figure 2.6: A skein relation to find the Jones polynomial of a Hopf link.

Notice that in Figure 2.6, the link in the middle is the same two unknots we calculated in 
Figure 2.5, and the last one is just a twisted unknot. Solving for $V_{L_{+}}$gives,

$$
V_{L_{+}}(t)=-t^{5 / 2}-t^{1 / 2}
$$
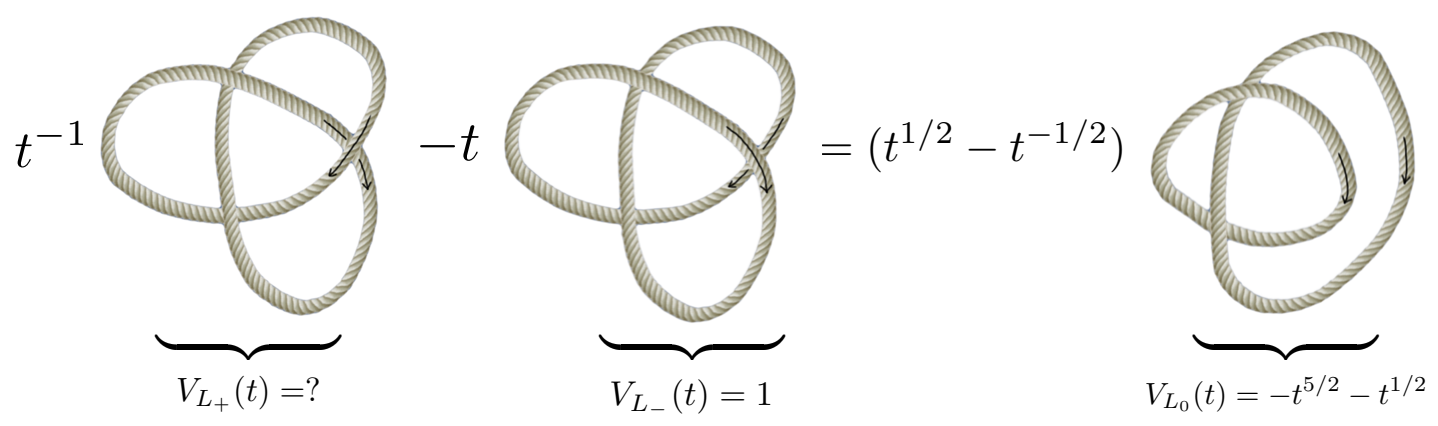

Figure 2.7: A skein relation to find the Jones polynomial of a right-handed trefoil knot.

Now we have everything we need to calculate the Jones polynomial for the trefoil knot, which is the first term in Figure 2.7. The middle knot in Figure 2.7 is an unknot and the last link is the Hopf link that we calculated with the previous skein relation. Solving this skein relation for $V_{L_{+}}$we get,

$$
V_{\text {trefoil }}(t)=t+t^{3}-t^{4}
$$

\subsubsection{The HOMFLY Polynomial}

There is a generalized version of the Jones polynomial, called the "HOMFLY" polynomial. This polynomial was discovered by Jim Hoste, Adrian Ocneanu, Kenneth Millett, Peter J. Freyd, W. B. R. Lickorish, and David N. Yetter [46]. The HOMFLY polynomial $S_{L}(\beta, z)$ satisfies the "generalized" skein relations 


$$
\begin{gathered}
S_{\hat{L}_{+}}=\alpha S_{\hat{L}_{0}}, \\
S_{\hat{L}_{-}}=\alpha^{-1} S_{\hat{L}_{0}}, \\
\beta S_{L_{+}}-\beta^{-1} S_{L_{-}}=z S_{L_{0}} .
\end{gathered}
$$

Here, $\beta$ is a parameter analogous to $t$ in 2.68 . $z$ and $\alpha$ are functions of $\beta$. The normalization condition is $S_{0}=1$ for the unknot, similar to the Jones polynomial. However, the knot diagrams for $\hat{L}$ differ from Figure 2.4. The correct knot diagrams are given in Figure 2.8.
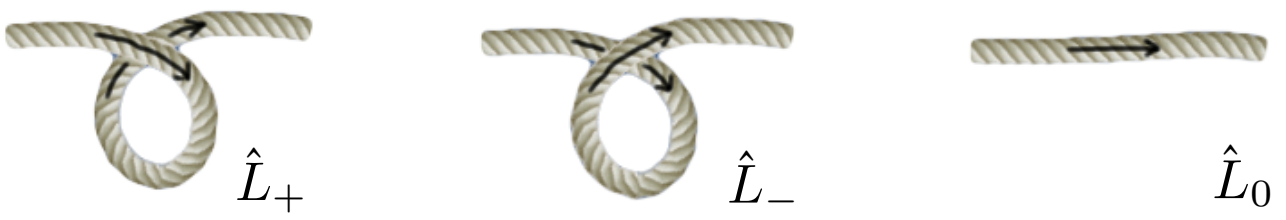

Figure 2.8: Knot diagrams for $\hat{L}$.

Although it looks like the diagrams for $\hat{L}_{+}$and $\hat{L}_{-}$are topologically the same in Figure 2.8 . they are not necessarily. Other parts of the knot can go though the loop, which is not shown in the figure. Solving 2.72 is very similar to solving (2.68) as shown in the example in subsection 2.10.2.

In the next section we will derive generalized skein relations for Wilson loop expectation values in Chern-Simons theory.

\subsection{Chern-Simons Theory and Link Invariants}

After Atiyah's proposal of the problem in 1988 [45], Witten found the meaning of the Jones polynomial in quantum field theory. Using 2D CFTs [1], he showed that Chern-Simons theory Wilson loop expectation values are link invariants. But here, for pedagogical reasons, 
we will review Cotta-Ramusino et al.'s work [2] that used only 3D field theory techniques.

As we defined earlier, a link is a union of $n$ non-intersecting knots. In the physics language this is a product of Wilson loops

$$
\left\langle W_{R_{1}}\left(C_{1}\right) \ldots W_{R_{n}}\left(C_{n}\right)\right\rangle \equiv\langle W(L)\rangle
$$

where each loop is a knot.

To derive skein relations, we need to focus on crossings. $L_{+}, L_{-}$and $L_{0}$ differ by small variations of the path on the same crossing. Under such a variation at a point $y$, the matrix $U\left(x_{2}, x_{1}\right)=\mathcal{P} \exp \left(-\int_{C}^{x_{1}} A_{\mu} d x^{\mu}\right)$ changes by

$$
\delta U\left(x_{2}, x_{1}, C\right)=i U\left(x_{2}, y, C\right) F_{\mu \nu}^{a} \Sigma^{\mu \nu} t_{R}^{a} U\left(y, x_{1}, C\right)
$$

where $\Sigma_{\mu \nu}$ is the area element of the deformed region, label $R$ indicates representation and there is no sum over $\mu$ and $\nu$. Since we want to see how $\langle W(L)\rangle$ changes under a variation of the path, we would like to calculate the expectation value $\left\langle F_{\mu \nu}^{a}(y) O_{1} O_{2} \ldots\right\rangle$. Using 2.10 , we can replace $F_{\mu \nu}$ by

$$
F_{\mu \nu}^{a}=\frac{4 \pi}{k} \epsilon_{\alpha \mu \nu} \frac{\delta S_{C S}}{\delta A_{\alpha}^{a}}
$$

With $Z=\int \mathcal{D} A e^{i S_{C S}}$, we write

$$
\left\langle F_{\mu \nu}^{a}(y) O_{1} O_{2} \ldots\right\rangle=\frac{1}{Z} \int \mathcal{D} A e^{i S_{C S}} F_{\mu \nu}^{a}(y) O_{1} O_{2} \ldots
$$

Using (2.75), the expectation value can be written as

$$
\left\langle F_{\mu \nu}^{a}(y) O_{1} O_{2} \ldots\right\rangle=-i \frac{4 \pi}{k} \frac{1}{Z} \int \mathcal{D} A \epsilon_{\alpha \mu \nu} \frac{\delta e^{i S_{C S}}}{\delta A_{\alpha}^{a}} O_{1} O_{2} \ldots
$$


After integrating by parts, the expectation value becomes

$$
\left\langle F_{\mu \nu}^{a}(y) O_{1} O_{2} \ldots\right\rangle=i \frac{4 \pi}{k} \frac{1}{Z} \int \mathcal{D} A e^{i S_{C S}} \epsilon_{\alpha \mu \nu} \frac{\delta}{\delta A_{\alpha}^{a}}\left(O_{1} O_{2} \ldots\right) .
$$

Now, using (2.74) and (2.78), we can calculate how $\left\langle\ldots U\left(x_{2}, x_{1}\right) \ldots\right\rangle$ changes under a small variation of the path. The result is

$$
\delta\left\langle\ldots U\left(x_{2}, x_{1}\right) \ldots\right\rangle=-i \frac{4 \pi}{k} \delta^{3}(x-y) \epsilon_{\alpha \mu \nu} \Sigma^{\mu \nu} d y^{\alpha}\left\langle\ldots U\left(x_{2}, x\right) t_{R}^{a} t_{R}^{a} U\left(y, x_{1}\right) \ldots\right\rangle .
$$

The factor in front of the term on the right hand side plays the key role in obtaining skein relations. Without the constants, the factor is $v=\delta^{3}(x-y) \epsilon_{\alpha \mu \nu} \Sigma^{\mu \nu} d y^{\alpha}$ and it can only take values $0, \pm 1 . d y^{\alpha}$ is tangent to the path and its orientation with respect to the plane defined by $\Sigma^{\mu \nu}$ decides the value of $v$. If $d y^{\alpha}$ is on the $\Sigma^{\mu \nu}$ plane, then the Levi-Civita tensor will take the value zero, since two of the indices will be the same. When $d y^{\alpha}$ is perpendicular to the plane, then depending on the direction $v$ takes values \pm 1 . This leads to three possible deformations of the a $\operatorname{link} L$, as

$$
\delta\left\langle W_{R}(L)\right\rangle=0
$$

and

$$
\delta\left\langle W_{R}(L)\right\rangle= \pm i \frac{4 \pi}{k} c_{R}\left\langle W_{R}(L)\right\rangle
$$

where $c_{R} \mathbb{1}=\sum_{a} t_{R}^{a} t_{R}^{a}$ is the quadratic Casimir in representation $R$. To cross over or under, the deformation has to be perpendicular to the plane defined by $\Sigma^{\mu \nu}$. Thus, in Figure 2.8, $L_{+}$and $L_{-}$corresponds to $d y^{\alpha}$ being perpendicular to the plane, which is given by (2.81). The case where $d y^{\alpha}$ belongs to the plane is given by 2.80 . 
The expectation values satisfy the following recursive relation,

$$
\left\langle W\left(\hat{L}_{+}\right)\right\rangle-\left\langle W\left(\hat{L}_{-}\right)\right\rangle=-i \frac{4 \pi}{k} c_{R}\left\langle W\left(\hat{L}_{0}\right)\right\rangle
$$

Since our goal is to obtain 2.72 , we write

$$
\left\langle W\left(\hat{L}_{+}\right)\right\rangle=\alpha\left\langle W\left(\hat{L}_{0}\right)\right\rangle \text { and }\left\langle W\left(\hat{L}_{-}\right)\right\rangle=\alpha^{-1}\left\langle W\left(\hat{L}_{0}\right)\right\rangle
$$

Solving 2.82) using 2.83 gives

$$
\alpha=1-i \frac{2 \pi}{k} c_{R}+\mathcal{O}\left(\frac{1}{k^{2}}\right)
$$

Deforming with an infinitesimally small circular area, an under-crossing can be related to an over-crossing as shown in Figure 2.9.
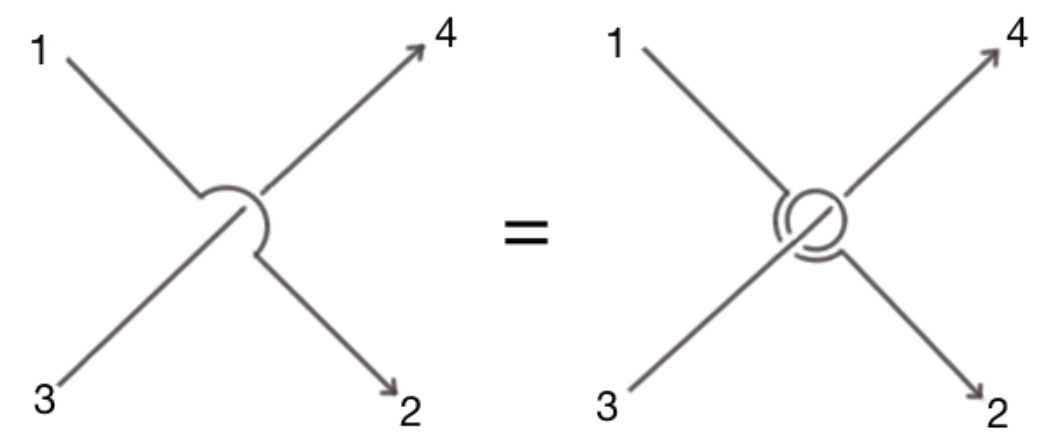

Figure 2.9: Relating under-crossings with over-crossings.

In terms of $W\left(L_{+}\right)$and $W\left(L_{-}\right)$, this deformation can be written as

$$
\left\langle W\left(L_{+}\right)\right\rangle=\left\langle W\left(L_{-}\right)\right\rangle+\left\langle\ldots U(1, x) i \Sigma^{\mu \nu} F_{\mu \nu}^{a} t_{R}^{a} U(x, 2) \ldots U(3,4) \ldots\right\rangle .
$$

Once again, we can replace $F_{\mu \nu}$ using (2.75). Then the functional derivative will act on both $1 \rightarrow 2$ and $3 \rightarrow 4$ paths in the last term. For the path $1 \rightarrow 2, d y^{\alpha}$ is on the plane defined by 
$\Sigma^{\mu \nu}$ which leads to the option 2.80 . Then, we get

$$
\left\langle W\left(L_{+}\right)\right\rangle=\left\langle W\left(L_{-}\right)\right\rangle-i \frac{4 \pi}{k} \sum_{a}\left\langle\ldots U(1, x) t_{R}^{a} U(x, 2) \ldots U(3, x) t_{R}^{a} U(x, 4) \ldots\right\rangle
$$

To get a recursive skein-like relation, the last term needs to be related to $W\left(L_{0}\right)$. The simplest way of doing this is choosing $t_{R}=t_{R}^{\prime}=t_{F}$, where $t_{F}$ is in fundamental representation. Now, we can use the Fierz identity for SU(N),

$$
\sum_{a} t_{i j}^{a} t_{k l}^{a}=\frac{1}{2} \delta_{i l} \delta_{j k}-\frac{1}{2 N} \delta_{i j} \delta_{k l}
$$

Then, 2.86 becomes

$$
\left\langle W\left(L_{+}\right)\right\rangle=\left(1+i \frac{2 \pi}{k} \frac{1}{N}\right)\left\langle W\left(L_{-}\right)\right\rangle-i \frac{2 \pi}{k}\left\langle W\left(L_{-}\right)\right\rangle .
$$

This recursive relation can be solved without introducing any new restrictions on $k$. But to have the skein relation

$$
\beta\left\langle W\left(L_{+}\right)\right\rangle-\beta^{-1}\left\langle W\left(L_{-}\right)\right\rangle=z(\beta)\left\langle W\left(L_{0}\right)\right\rangle,
$$

$k$ needs to be a large integer, with the following definitions:

$$
\beta=1-i \frac{2 \pi}{k} \frac{1}{2 N}+\mathcal{O}\left(\frac{1}{k^{2}}\right) \text { and } z=-i \frac{2 \pi}{k}+\mathcal{O}\left(\frac{1}{k^{2}}\right)
$$

Equation 2.89), together with (2.83), show that Chern-Simons theory Wilson loop expectation values satisfy 2.72 . Equations 2.90 relate the skein parameters to the physical constants of Chern-Simons theory. With these solutions, we have shown that Wilson loop expectation values of Chern-Simons theory are link invariants that can be obtained by solving 2.89), with the knot diagrams given in Figure 2.4. 


\subsection{Geometric Quantization}

In geometric quantization ${ }^{2}$, one starts with taking the phase space of a classical theory and constructs a pre-quantum Hilbert space. This step prepares the phase space for quantization and it is not necessary in other quantization methods, like path integral formulation or canonical quantization using creation and annihilation operators. In the prequantum Hilbert space, wave-functions depend on all phase space variables; while in the quantum theory, they should depend on half of them that commute with each other. This feature makes the pre-quantum Hilbert space too large to describe a real quantum system. Thus, a reduction of the pre-quantum Hilbert space becomes necessary.

Quantization of a classical theory replaces the algebra of Poisson brackets with the algebra of commutation rules for quantum operators. The quantization process should lead to a Hilbert space that provides an irreducible representation of the operator algebra. This is done by choosing a subspace of the pre-quantum Hilbert space, which forces the wavefunctions to depend on half of the phase space variables that commute. This ensures that quantum wave-functions are not simultaneous eigenstates of non-commuting observables. There are three general ways of doing this Hilbert space reduction: choosing the position Hilbert space, the momentum Hilbert space or the Segal-Bargmann space. This reduction procedure is called choosing a polarization for the wave-functions. This is done by choosing a direction in the phase space that leaves the wave-function constant. It is called polarization due to its analogous nature compared to the polarization of electromagnetic waves.

\footnotetext{
${ }^{2}$ In this section, we will mostly follow refs. 33.47
} 


\subsubsection{Phase Space Geometry}

Before we get into the details about polarizations, we shall discuss how to obtain the geometry of the phase space from a given Lagrangian. Phase space is a smooth even dimensional manifold that will be denoted as $\mathcal{M}$. We will start with varying the action $S=\int d^{4} x \mathcal{L}$ with a general Lagrangian of a spin 1 gauge field. The variation of the Lagrangian is

$$
\begin{aligned}
\delta \mathcal{L} & =\frac{\partial \mathcal{L}}{\partial A_{\mu}} \delta A_{\mu}+\frac{\partial \mathcal{L}}{\partial\left(\partial_{\mu} A_{\nu}\right)} \partial_{\mu} \delta A_{\nu} \\
& =\left[\frac{\partial \mathcal{L}}{\partial A_{\mu}}-\partial_{\nu} \frac{\partial \mathcal{L}}{\partial\left(\partial_{\nu} A_{\mu}\right)}\right] \delta A_{\mu}+\partial_{\nu}\left(\frac{\partial \mathcal{L}}{\partial\left(\partial_{\nu} A_{\mu}\right)} \delta A_{\mu}\right) .
\end{aligned}
$$

It is standard to assume that either $\delta A_{\mu}$ or $\frac{\partial \mathcal{L}}{\partial\left(\partial_{\nu} A_{\mu}\right)}$ vanishes at the spatial boundary $\partial V$; but assuming that it vanishes at time boundary $t_{i}$ and $t_{f}$ depends on the quantization method. In canonical or geometric quantization, this second assumption is not forced. Instead, the phase space geometry is obtained from the boundary term of 2.91 , since it is a function of only the phase space variables $A_{\mu}$ and their conjugate momenta $\Pi^{\mu}$.

The boundary term from the action is

$$
\int_{t_{i}}^{t_{f}} d t \int_{V} d^{3} x \frac{\partial \mathcal{L}}{\partial\left(\partial_{0} A_{\mu}\right)} \delta A_{\mu}
$$

The integrand of the time integral is called the symplectic potential. Since $\Pi^{\mu}=\frac{\partial \mathcal{L}}{\partial\left(\partial_{0} A_{\mu}\right)}$ we can write the symplectic potential as

$$
\mathscr{A}=\int d^{3} x \Pi^{\mu} \delta A_{\mu} .
$$

Here $\delta$ is the exterior differentiation in the phase space, thus it satisfies the Poincare lemma 
$\delta^{2}=0$. The symplectic two-form is given by $\Omega=\delta \mathscr{A}$ thus,

$$
\Omega=\int d^{3} x \delta \Pi^{\mu} \delta A_{\mu} .
$$

$\Omega$ plays the role of a metric and it defines the geometry of the phase space. The Poisson brackets can be obtained from $\Omega^{-1}$ as

$$
\{f, g\}=\Omega^{\mu \nu} \partial_{\mu} f \partial_{\nu} g
$$

where $f$ and $g$ are functions that live on $\mathcal{M}$.

Symplectic two-form is invariant under canonical transformations. But symplectic potential transforms as $\mathscr{A}^{\prime} \rightarrow \mathscr{A}+\delta \Lambda$, where $\Lambda$ is some function of the phase space variables. In some sense $\mathscr{A}$ transforms like a $U(1)$ gauge field.

\subsubsection{Pre-quantization}

For functions $f$ and $g$ that live on $\mathcal{M}$, we introduce the corresponding pre-quantum operators $Q(f)$ and $Q(g)$ by

$$
Q_{\text {pre }}(\{f, g\})=\frac{1}{i \hbar}\left[Q_{\text {pre }}(f), Q_{\text {pre }}(g)\right] .
$$

This equation is solved by

$$
Q_{\text {pre }}(f)=i \hbar\left(X_{f}-\frac{i}{\hbar} \mathscr{A}_{j} X_{f}^{j}\right)+f
$$

where $\mathscr{A}_{j}$ are components of the symplectic potential and $X_{f}$ is a Hamiltonian vector field given by

$$
X_{f}=\frac{\partial f}{\partial x_{i}} \frac{\partial}{\partial p_{i}}-\frac{\partial f}{\partial p_{i}} \frac{\partial}{\partial x_{i}} .
$$


To describe a single point particle, we can choose $f=x_{i}$ and $g=p_{i}$. Then the components of vector $X$ are

$$
X_{x_{i}}=\frac{\partial}{\partial p_{i}} \text { and } X_{p_{i}}=-\frac{\partial}{\partial x_{i}}
$$

Using $\mathscr{A}=p_{i} d x_{i}$, we get

$$
Q_{\text {pre }}\left(x_{i}\right)=x_{i}+i \hbar \frac{\partial}{\partial p_{i}} \text { and } Q_{p r e}\left(p_{i}\right)=-i \hbar \frac{\partial}{\partial x_{i}} .
$$

These operators satisfy $\left[Q_{\text {pre }}\left(x_{i}\right), Q_{\text {pre }}\left(p_{i}\right)\right]=i \hbar$; thus, 2.100 is a representation. But it is not irreducible. This problem comes from pre-quantum Hilbert space being too large. The solution is to choose a subspace of the pre-quantum Hilbert space and this is done by polarizing the wave-function. The pre-quantum wave-function is a function of all phase space variables, i.e. $\Phi=\Phi\left(x_{i}, p_{i}\right)$. Polarization forces the quantum wave-function to depend on half of the phase space variables that commute. There are three general ways of doing this: choosing the position Hilbert space where the quantum wave-function $\psi=\psi\left(x_{i}\right)$, choosing the momentum Hilbert space where $\psi=\psi\left(p_{i}\right)$, or choosing the Segal-Bargmann space where $\psi=\psi(z)$ and $z=x+i p$. The first two options are obtained with real polarizations and the last one is with holomorphic polarization.

The polarization condition is defined by

$$
\mathcal{D}_{i} \Phi=0
$$

where $\mathcal{D}_{i}$ is a covariant derivative given by $\mathcal{D}_{i}=\partial_{i}-i \mathscr{A}_{i}$. Then, the curvature is given by the commutator $\left[\mathcal{D}_{i}, \mathcal{D}_{j}\right]=i \Omega_{i j}$.

For a polarization to be well defined, the wave-function has to be square integrable. Otherwise a well define inner product cannot be defined. The pre-quantum inner product is 
given by

$$
\langle 1 \mid 2\rangle=\int d \sigma(\mathcal{M}) \Phi_{1}^{*} \Phi_{2}
$$

where integration is done over all phase space variables. Under canonical transformation $\mathscr{A}^{\prime} \rightarrow \mathscr{A}+\delta \Lambda$ the pre-quantum wave functional transforms like a matter field, as $\Phi^{\prime} \rightarrow e^{i \Lambda} \Phi$. Thus, 2.102 is invariant under canonical transformations, as one would expect.

\subsubsection{Real Polarizations}

For a point particle in one dimension, symplectic two-form is given by,

$$
\Omega=d p \wedge d x
$$

and up to a canonical transformation, the symplectic potential is

$$
\mathscr{A}=p d x
$$

As we have shown, the pre-quantum operators for position $\mathcal{Q}_{\text {pre }}(x)$ and momentum $\mathcal{Q}_{\text {pre }}(p)$ should satisfy

$$
\left[\mathcal{Q}_{\text {pre }}(x), \mathcal{Q}_{\text {pre }}(p)\right]=i \hbar
$$

One representation that satisfies this commutator is given by

$$
\mathcal{Q}_{\text {pre }}(x)=i \hbar \frac{\partial}{\partial p}+x \text { and } \mathcal{Q}_{p r e}(p)=-i \hbar \frac{\partial}{\partial x}
$$

In $(x, p)$ coordinates, there are two polarization choices:

$$
\mathcal{D}_{p} \Phi(x, p)=0 \text { or } \mathcal{D}_{x} \Phi(x, p)=0 .
$$


For $\mathscr{A}=p d x, \mathcal{D}_{p}=\partial_{p}$ and $\mathcal{D}_{x}=\partial_{x}-i p$. Choosing the first polarization condition gives $\Phi(x, p)=\psi(x)$, where $\psi$ is the quantum wave-function. For this case, the pre-quantum operators are

$$
\mathcal{Q}_{\text {pre }}(x)=x \text { and } \mathcal{Q}_{\text {pre }}(p)=-i \hbar \frac{\partial}{\partial x}
$$

This is an irreducible representation. Choosing the second condition leads to $\Phi(x, p)=$ $\psi(p) e^{i p x}$. One can always do a canonical transformation, such as

$$
\mathscr{A} \rightarrow \mathscr{A}^{\prime}=\mathscr{A}-d(x p)=-x d p
$$

to obtain $\mathcal{D}_{p}=\partial_{p}+i x$ and $\mathcal{D}_{x}=\partial_{x}$. Then, choosing $\mathcal{D}_{x} \Phi=0$ gives $\Phi(x, p)=\psi(p)$ and quantum operators for this case are

$$
\mathcal{Q}_{\text {pre }}(x)=i \hbar \frac{\partial}{\partial p} \text { and } \mathcal{Q}_{\text {pre }}(p)=p
$$

For all of these cases, $\psi$ will depend on either $x$ or $p$.

In real polarizations, the pre-quantum inner product

$$
\langle\psi \mid \psi\rangle=\int d x d p \psi^{*}(x) \psi(x)
$$

is not finite. Thus, it cannot be used as the quantum inner product. The irrelevant degrees of freedom should not be integrated over. If the chosen subspace is the position Hilbert space, then integration should be over just $d x_{i}$ (or $d p_{i}$ for the momentum Hilbert space) as,

$$
\langle\psi \mid \psi\rangle=\int d x \psi^{*}(x) \psi(x) \text { or }\langle\psi \mid \psi\rangle=\int d p \psi^{*}(p) \psi(p)
$$

It is well known that these two cases are physically equivalent. The equivalence can be shown using the fact that $\psi(x)$ and $\psi(p)$ are Fourier transforms of each other. But just not 
integrating over $d p$ in the position Hilbert space (or $d x$ in the momentum Hilbert space) is not the proper way of defining a finite inner product. The correct way of doing this is called the half-form quantization.

\subsubsection{Half-Form Quantization}

A half-form is the square root of a one-form, defined as

$$
\sqrt{d x} \otimes \sqrt{d x}=d x
$$

To define a finite inner product, instead of using $\psi$ and integral measure as separate objects, a composite object $s=\psi \otimes \sqrt{d x}$ needs to be defined as a polarized section. Then the inner product is given by

$$
\|s\|^{2}=\int|\psi|^{2} d x .
$$

This procedure makes sure that the integration is done over only the relevant degree of freedom and the inner product is finite.

In half-form quantization, quantum operators act on $s$, not just $\psi$. An operator $Q(f)$ on the half-form space acting on $s$ is given by 47

$$
Q(f) s=\left(Q_{\text {pre }}(f) \psi\right) \otimes \sqrt{d z}-i \hbar \psi \otimes \mathcal{L}_{X_{f}} \sqrt{d z}
$$

where $\mathcal{L}$ is a Lie derivative. The second term in 2.115 is called the "metaplectic correction". 


\subsubsection{Complex Polarizations}

When the phase space is Kähler, it is advantageous to work with complex coordinates.

Introducing the local complex coordinates $z^{a}, \bar{z}^{a}$, we can write

$$
\Omega=\frac{1}{2} \Omega_{a \bar{a}} d z^{a} \wedge d \bar{z}^{\bar{a}}
$$

The metric is given by

$$
g_{a \bar{b}}=\partial_{a} \partial_{\bar{b}} K
$$

where $\mathrm{K}$ is a real function,n called the Kähler potential. Metric components and symplectic two-form components are related by $\Omega_{a \bar{b}}=-\Omega_{\bar{b} a}=i g_{a \bar{b}}$. The symplectic two-form can also be written as

$$
\Omega=i \partial \bar{\partial} K
$$

where $\partial=d z^{a} \wedge \frac{\partial}{\partial z^{a}}$ and $\bar{\partial}=d \bar{z}^{\bar{a}} \wedge \frac{\partial}{\partial \bar{z}^{\bar{a}}}$.

With the Kähler potential, two covariant derivatives can be defined as

$$
\mathcal{D}_{a}=\partial_{a}-i \mathscr{A}_{a} \text { and } \mathcal{D}_{\bar{a}}=\partial_{\bar{a}}-i \mathscr{A}_{\bar{a}}
$$

where the connections are given by

$$
\mathscr{A}_{a}=-\frac{i}{2} \partial_{a} K \text { and } \mathscr{A}_{\bar{a}}=\frac{i}{2} \partial_{\bar{a}} K
$$

These are the components of the symplectic potential that is given by $\Omega=d \mathscr{A}$. Thus, the commutator of the covariant derivatives in 2.119 satisfy $\left[\mathcal{D}_{a}, \mathcal{D}_{\bar{a}}\right]=i \Omega_{a \bar{a}}$.

With the covariant derivatives defined above, two polarization conditions can be written: $\mathcal{D}_{\bar{a}} \Phi=0$ and $\mathcal{D}_{a} \Phi=0$. The first one

$$
\mathcal{D}_{\bar{a}} \Phi=\left(\partial_{\bar{a}}+\frac{1}{2} \partial_{\bar{a}} K\right) \Phi=0
$$


is solved by

$$
\Phi\left(z^{a}, \bar{z}^{a}\right)=e^{-\frac{1}{2} K\left(z^{a}, \bar{z}^{a}\right)} \Psi\left(z^{a}\right)
$$

where $\Psi$ is holomorphic in $z^{a}$. In holomorphic polarizations, the pre-quantum inner product can be retained at the quantum level as,

$$
\left\langle\Phi_{1} \mid \Phi_{2}\right\rangle=\int d \sigma(\mathcal{M}) \Phi_{1}^{*} \Phi_{2} \longrightarrow\left\langle\psi_{1} \mid \psi_{2}\right\rangle=\int d \sigma(\mathcal{M}) e^{-K} \psi_{1}^{*} \psi_{2}
$$

This inner product is well behaved only if $K$ is positively defined. For $K<0, \mathcal{D}_{a} \Phi=0$ has to be chosen. Clearly, only one of the two polarizations will give a well behaved inner product. Hence, the appropriate one must be chosen.

One important point that distinguishes the holomorphic polarization from real polarizations is the integral measure. In real polarizations, integration is done only over the polarized section. However, in holomorphic polarizations, the integration is done over the whole phase space volume. $\psi$ might just depend on $z$, but $\psi^{*}$ will have $\bar{z}$ dependence and $K$ always depends on both $z$ and $\bar{z}$. Thus, integration over both $z$ and $\bar{z}$ is necessary. This feature seem to make half-from quantization unnecessary for holomorphic polarizations. But there still are advantages in using half-forms, even though the inner product is already finite without using them.

The most well known example for holomorphic quantization is the coherent state quantization of a simple harmonic oscillator. The Hamiltonian for this system is given by

$$
H=\frac{1}{2 m}\left(p^{2}+(m \omega x)^{2}\right)=\frac{1}{2 m}\left(p^{2}+y^{2}\right)
$$

where $y=m \omega x$. The symplectic potential is

$$
\mathscr{A}=\frac{1}{2}(p d x-x d p)=\frac{1}{2 m \omega}(p d y-y d p) .
$$


Using (2.97) with $f=H$, we get

$$
Q_{p r e}(H)=i \hbar\left(y \frac{\partial}{\partial p}-p \frac{\partial}{\partial y}\right)
$$

Now, we switch to complex coordinates

$$
z=\frac{1}{m \omega}(y-i p) \text { and } \bar{z}=\frac{1}{m \omega}(y+i p) .
$$

Then, choosing the polarization condition

$$
D_{\bar{z}} \Phi(z, \bar{z})=\left(\partial_{\bar{z}}+\frac{m \omega}{2 \hbar} z\right) \Phi(z, \bar{z})=0
$$

gives

$$
\Phi(z, \bar{z})=\psi(z) e^{-\frac{m \omega}{2 \hbar} z \bar{z}}
$$

and $K=\frac{m \omega}{\hbar} z \bar{z}$ is the Kähler potential. Now, we can write

$$
Q_{\text {pre }}(H) \psi=\hbar\left(y \frac{\partial}{\partial p}-p \frac{\partial}{\partial y}\right) \psi\left(\frac{1}{m \omega}(y-i p)\right)=\hbar \omega z \frac{d \psi}{d z}
$$

$\psi$ is in the form $[47]$ of $\psi=\sum_{n} a_{n} z^{n}$. Thus, $z \frac{d \psi}{d z}=n \psi$ and this gives the spectrum

$$
E_{n}=\hbar \omega n
$$

This differs from the correct spectrum of the harmonic oscillator by $\hbar \omega / 2$. To obtain the correct spectrum, the system needs to be quantized with half-forms.

For $f=H$, the Lie derivative acting on $\sqrt{d z}$ in the metaplectic correction term is given by 47

$$
\mathcal{L}_{X_{H}} \sqrt{d z}=\frac{i \omega}{2} \sqrt{d z}
$$


Then using 2.115, we can write

$$
Q(H) s=\hbar \omega n \psi \otimes \sqrt{d z}+\frac{\hbar \omega}{2} \psi \otimes \sqrt{d z}
$$

From this equation, it can be seen that the missing $\hbar \omega / 2$ in the spectrum appears as a metaplectic correction, giving the correct spectrum

$$
E_{n}=\hbar \omega\left(n+\frac{1}{2}\right) .
$$

Although holomorphic polarizations lead to a well defined quantization even without the help of half-forms, in some cases like this, it can be advantageous to do half-form quantization. However, we will not use half-forms for Chern-Simons theory, since there seems to be no natural way of doing so.

\subsubsection{Geometric Quantization of Gauge Theories}

In quantum mechanics, solving Schrödinger's equation is sufficient to find the wavefunction. However, in a gauge field theory, Gauss' law also needs to be solved in addition to Schrödinger's equation.

Gauss' law operator is the generator of infinitesimal gauge transformations of a given gauge theory. In $S U(N)$ gauge theories, the gauge field transforms as

$$
A_{\mu} \rightarrow A_{\mu}^{g}=g A_{\mu} g^{-1}-\left(\partial_{\mu} g\right) g^{-1}
$$

where $g=\exp \left(-i t^{a} \theta^{a}\right)$. For $\theta \ll 1$, the infinitesimal transformation $\left(g \approx 1-i t^{a} \theta^{a}\right)$ can be written as

$$
A_{\mu}^{g}=A_{\mu}+i t^{a}\left(D_{\mu} \theta\right)^{a} .
$$


Infinitesimal transformations are generated by the vector field

$$
\xi=\int d^{n} x \delta A_{\mu}^{a} \frac{\delta}{\delta A_{\mu}^{a}}=-\int d^{n} x\left(D_{\mu} \theta\right)^{a} \frac{\delta}{\delta A_{\mu}^{a}},
$$

where $n$ is the dimension of the space of gauge fields. To find the Gauss' law operator, we write the interior contraction of the symplectic two-form with the vector field,

$$
i_{\xi} \Omega=\xi^{\mu} \Omega_{\mu \nu} d q^{\nu}
$$

where $q^{\nu}$ is a phase space coordinate. Generally, for transformations in the phase space, the interior contraction satisfies

$$
i_{\xi} \Omega=-\delta f
$$

where $f$ is some function of the phase space variables. For infinitesimal gauge transformations, $f=\int G^{a} \theta^{a}$ and then 2.139 becomes

$$
i_{\xi} \Omega=-\delta \int d^{n} x G^{a} \theta^{a}
$$

Here $G^{a}$ is the Gauss' law operator and the Gauss' law is given by

$$
G^{a} \psi=0
$$

$G^{a}=0$ also appears as one of the equations of motion.

At least for the gauge theories that we are interested in, such as CS, YM or TMYM, the wave-functional can be factorized in the form $\psi=\phi \chi$. Here, $\phi$ is the part that satisfies Gauss' law constraint $G^{a} \Psi=0$ and $\chi$ is the gauge invariant part that is necessary for $\psi$ to satisfy the Schrödinger's equation $\mathcal{H} \psi=\mathcal{E} \psi$. To find $\phi$, the standard technique is to make an infinitesimal gauge transformation on $\psi$, then force the Gauss' law to obtain a condition 
that is usually solved by some Wess-Zumino-Witten(WZW) action. Once $\phi$ is known, then the Schrödinger's equation can be tackled to find $\chi$. In $2+1$ dimensional gauge theories, $\chi$ is where the scale dependence is hidden. 


\section{CHAPTER 3 \\ GEOMETRIC QUANTIZATION OF CHERN-SIMONS THEORY}

In this chapter, we will review the geometric quantization of non-Abelian ChernSimons(CS) theory, mostly following Bos and Nair's work 33,38

The CS action is given by

$$
S_{C S}=-\frac{k}{4 \pi} \int_{\Sigma \times\left[t_{i}, t_{f}\right]} d^{3} x \epsilon^{\mu \nu \alpha} \operatorname{Tr}\left(A_{\mu} \partial_{\nu} A_{\alpha}+\frac{2}{3} A_{\mu} A_{\nu} A_{\alpha}\right),
$$

where $\Sigma$ is an orientable two dimensional surface. This action is classically not gauge invariant, but in the quantum theory it can be made gauge invariant by restricting $k$ to be an integer, as discussed in section 2.4. The equations of motion for this theory are

$$
F_{\mu \nu}=\partial_{\mu} A_{\nu}-\partial_{\nu} A_{\mu}+\left[A_{\mu}, A_{\nu}\right]=0 .
$$

In the temporal gauge $A_{0}=0$ with complex coordinates $A_{z}=\frac{1}{2}\left(A_{1}+i A_{2}\right)$ and $A_{\bar{z}}=\frac{1}{2}\left(A_{1}-i A_{2}\right)$, the CS action becomes

$$
S_{C S}=-\frac{i k}{2 \pi} \int d t d \mu_{\Sigma} \operatorname{Tr}\left(A_{\bar{z}} \partial_{0} A_{z}-A_{z} \partial_{0} A_{\bar{z}}\right)
$$

The equations of motion $\left(F_{\mu \nu}=0\right)$ in this gauge makes $A_{z}$ and $A_{\bar{z}}$ time independent along with constraining $F_{z \bar{z}}=0$. A very important feature is that the conjugate momenta are given by the gauge fields,

$$
\Pi^{a z}=\frac{i k}{4 \pi} A_{\bar{z}}^{a} \text { and } \Pi^{a \bar{z}}=-\frac{i k}{4 \pi} A_{z}^{a} .
$$

Later, we will see a similar behavior in TMYM theory which is crucial for our work.

${ }^{1}$ Another comprehensive discussion on this subject can be found in ref. 48 
Following the method outlined in subsection 2.12.1, we write the symplectic potential

as

$$
\mathscr{A}=\frac{i k}{4 \pi} \int_{\Sigma}\left(A_{\bar{z}}^{a} \delta A_{z}^{a}-A_{z}^{a} \delta A_{\bar{z}}^{a}\right)
$$

The symplectic two-form of CS theory is given by $\Omega=\delta \mathscr{A}$,

$$
\Omega=\frac{i k}{2 \pi} \int_{\Sigma} \delta A_{\bar{z}}^{a} \delta A_{z}^{a}
$$

This phase space is Kähler and the Kähler potential is given by $\Omega=i \partial \bar{\partial} K$, where $\partial=$ $\delta A_{z}^{a} \wedge \frac{\partial}{\partial A_{z}^{a}}$ and $\bar{\partial}=\delta A_{\bar{z}}^{a} \wedge \frac{\partial}{\partial A_{\bar{z}}^{a}} ;$ hence,

$$
K=\frac{k}{2 \pi} \int_{\Sigma} A_{\bar{z}}^{a} A_{z}^{a}
$$

As we discussed in section 2.8, in simply connected spaces it is possible to parametrize the gauge fields as

$$
A_{\bar{z}}=-\partial_{\bar{z}} U U^{-1} \text { and } A_{z}=U^{\dagger-1} \partial_{z} U^{\dagger}
$$

Here, $\mathrm{U}$ is a complex $\mathrm{SL}(\mathrm{N}, \mathbb{C})$ matrix which transforms like $U^{g}=g U$ where $g \in \mathcal{G}$ and $\mathcal{G}$ is the gauge group. We will continue with taking $\mathcal{G}=S U(N) . U$ is given by

$$
U(x, 0, C)=\mathcal{P} \exp \left(-\int_{C}^{x}\left(A_{\bar{z}} d \bar{z}+\mathcal{A}_{z} d z\right)\right)
$$

where $\mathcal{A}_{z}$ satisfies $\partial_{z} A_{\bar{z}}-\partial_{\bar{z}} \mathcal{A}_{z}+\left[\mathcal{A}_{z}, A_{\bar{z}}\right]=0$ and this flatness condition makes $U$ invariant under small deformations of the path $C$ on $\Sigma$. From $(3.9)$, it follows that

$$
\mathcal{A}_{z}=-\partial_{z} U U^{-1} \text { and } \mathcal{A}_{\bar{z}}=U^{\dagger-1} \partial_{\bar{z}} U^{\dagger}
$$




\subsection{Gauss' Law}

Infinitesimal gauge transformations $\left(g \approx 1-i t^{a} \varepsilon^{a}\right)$ are generated by the vector field given by 2.137). In complex coordinates, it is

$$
\xi=-\int_{\Sigma}\left[\left(D_{z} \varepsilon\right)^{a} \frac{\delta}{\delta A_{z}^{a}}+\left(D_{\bar{z}} \varepsilon\right)^{a} \frac{\delta}{\delta A_{\bar{z}}^{a}}\right]
$$

To find the Gauss' law, we look at the interior contraction

$$
\begin{aligned}
i_{\xi} \Omega & =-\frac{i k}{2 \pi} \int_{\Sigma} \varepsilon^{a}\left[-\left(D_{z} \delta A_{\bar{z}}\right)^{a}+\left(D_{\bar{z}} \delta A_{z}\right)^{a}\right] \\
& =-\delta\left(\frac{i k}{2 \pi} \int_{\Sigma} F_{z \bar{z}}^{a} \varepsilon^{a}\right) .
\end{aligned}
$$

Then using 2.140, we can write the Gauss' law operator for CS theory as

$$
G^{a}=\frac{i k}{2 \pi} F_{z \bar{z}}^{a}
$$

\subsection{The Wave-Functional}

We choose the holomorphic polarization, which makes the quantum wave-functional $\psi$ only $A_{\bar{z}}$ dependent. The pre-quantum and quantum wave-functionals are related by $\Phi\left[A_{z}, A_{\bar{z}}\right]=e^{-\frac{1}{2} K} \psi\left[A_{\bar{z}}\right]$, where $K$ is the Kähler potential given by (3.7). Since the phase space is Kähler, the pre-quantum inner product can be retained at the quantum level, as

$$
\langle 1 \mid 2\rangle=\int d \mu(\mathcal{M}) \Phi_{1}^{*} \Phi_{2} \rightarrow \int d \mu(\mathcal{M}) e^{-K} \psi_{1}^{*} \psi_{2}
$$

where $d \mu(\mathcal{M})$ is the Liouville measure defined by the symplectic two-form.

Upon quantization we can write,

$$
A_{z}^{a} \psi\left[A_{\bar{z}}^{a}\right]=\frac{2 \pi}{k} \frac{\delta}{\delta A_{\bar{z}}^{a}} \psi\left[A_{\bar{z}}^{a}\right] .
$$


Since no currents are present, the wave-functional must satisfy $F_{z \bar{z}} \psi\left[A_{\bar{z}}\right]=0$ which is the Gauss' law of CS theory. We then make an infinitesimal gauge transformation on the wave-functional $\psi$ with parameter $\varepsilon$,

$$
\begin{aligned}
\delta_{\varepsilon} \psi\left[A_{\bar{z}}\right] & =\int d^{2} z \delta_{\varepsilon} A_{\bar{z}}^{a} \frac{\delta \psi}{\delta A_{\bar{z}}^{a}} \\
& =\int d^{2} z \varepsilon^{a}\left(\partial_{\bar{z}} \frac{\delta}{\delta A_{\bar{z}}^{a}}+i f^{a b c} A_{\bar{z}}^{b} \frac{\delta}{\delta A_{\bar{z}}^{c}}\right) \psi \\
& =-\frac{k}{2 \pi} \int d^{2} z \varepsilon^{a}\left(F_{z \bar{z}}^{a}-\partial_{z} A_{\bar{z}}^{a}\right) \psi
\end{aligned}
$$

Then applying the Gauss' Law constraint $F_{z \bar{z}} \psi\left[A_{\bar{z}}\right]=0$ gives

$$
\delta_{\varepsilon} \psi\left[A_{\bar{z}}\right]=\frac{k}{2 \pi} \int d^{2} z \varepsilon^{a} \partial_{z} A_{\bar{z}}^{a} \psi\left[A_{\bar{z}}\right]
$$

From 2.49, we know that this condition is solved by writing

$$
\psi\left[A_{\bar{z}}\right]=\exp \left(k S_{W Z W}(U)\right)
$$

In general, the wave-functional in 3.18 can have a gauge invariant factor $\chi$ which can be found by solving the Schrodinger's equation $\mathcal{H} \psi=\mathcal{E} \psi$. But since the CS Hamiltonian for ground state is zero in the temporal gauge, we take $\chi=1 . \chi$ is where the scale dependence is hidden. For a topological theory like CS, a constant $\chi$ is expected.

\subsection{The Measure}

Using the symplectic two-form of CS theory (3.6), we can write the metric on $\mathfrak{A}$, the space of gauge potentials 37, as

$$
\begin{aligned}
d s_{\mathfrak{A}}^{2} & =\int d^{2} x \delta A_{i}^{a} \delta A_{i}^{a}=-8 \int \operatorname{Tr}\left(\delta A_{\bar{z}} \delta A_{z}\right) \\
& =8 \int \operatorname{Tr}\left[D_{\bar{z}}\left(\delta U U^{-1}\right) D_{z}\left(U^{\dagger-1} \delta U^{\dagger}\right)\right] .
\end{aligned}
$$


Our goal is to write the volume element of this space in terms of the volume of $S L(N, \mathbb{C})$, which has the Cartan-Killing metric

$$
d s_{S L(N, \mathbb{C})}^{2}=8 \int \operatorname{Tr}\left[\left(\delta U U^{-1}\right)\left(U^{\dagger-1} \delta U^{\dagger}\right)\right]
$$

The volumes of these two spaces are related by

$$
d \mu(\mathfrak{A})=\operatorname{det}\left(D_{\bar{z}} D_{z}\right) d \mu\left(U, U^{\dagger}\right)
$$

This measure is not gauge invariant. To make it invariant, we use the $S U(N)$ gauge invariant matrix $H=U^{\dagger} U$ that we defined in section 2.8, where $H \in S L(N, \mathbb{C}) / S U(N)$. Now, we can write

$$
d \mu(\mathfrak{A})=\operatorname{det}\left(D_{\bar{z}} D_{z}\right) d \mu(H)
$$

As shown in section 2.8, the determinant is

$$
\operatorname{det}\left(D_{\bar{z}} D_{z}\right)=\text { constant } \times e^{2 c_{A} S_{W Z W}(H)},
$$

where $c_{A}$ is the quadratic Casimir in the adjoint representation given by $c_{A} \delta^{a b}=f^{a m n} f^{b m n}$.

Now that we have the measure and the wave functional, we can write the inner product

$$
\left\langle\psi_{1} \mid \psi_{2}\right\rangle=\int d \mu(\mathfrak{A}) e^{-K} \psi_{1}^{*} \psi_{2}
$$

Using the Polyakov-Wiegmann identity we get,

$$
e^{-K} \psi^{*} \psi=e^{k S_{W Z W}(H)}
$$

Then, the inner product for $\psi$ becomes

$$
\langle\psi \mid \psi\rangle=\int d \mu(H) e^{\left(2 c_{A}+k\right) S_{W Z W}(H)} .
$$




\subsection{The $\Sigma=S^{1} \times S^{1}$ Case}

If the space is not simply connected, the parametrization we used in (3.8) needs modification. On a torus, as discussed in section 2.8, the correct parametrization is

$$
A_{\bar{z}}=-\partial_{\bar{z}} U U^{-1}+U i \pi(\operatorname{Im} \tau)^{-1} a U^{-1}
$$

where $\tau$ is the modular parameter of the torus and $a$ is a constant gauge field. The new term can be absorbed in a matrix as $V=U \exp \left[i \pi(\operatorname{Im} \tau)^{-1}(z-\bar{z}) a\right]$ and then $A_{\bar{z}}$ can be once again parametrized in the form $-\partial_{\bar{z}} V V^{-1}$. But this gives rise to a new factor in the wave-functional which depends on $a$. Now the wave-functional is

$$
\psi\left[A_{\bar{z}}\right]=\exp \left(k S_{W Z W}(V)\right) \Upsilon(a)
$$

Finding this new factor is not straightforward and we will not review its calculation here, but the result can be found in ref. 38 .

\subsection{Wilson Loops in Chern-Simons Theory}

The Wilson loop operator for representation $R$ and path $C$ is given by

$$
W_{R}(C)=\operatorname{Tr}_{R} \mathcal{P} e^{-\oint_{c} A_{\mu} d x^{\mu}}
$$

As shown in section 2.10, in CS theory, the expectation value of this operator can be calculated directly from skein relations without any field theory calculation. Up to some approximation, a generalized skein relation can be obtained [2] for WLEVs in the fundamental representation, as

$$
\beta\left\langle W_{L_{+}}\right\rangle-\beta^{-1}\left\langle W_{L_{-}}\right\rangle=z(\beta)\left\langle W_{L_{0}}\right\rangle
$$


where

$$
\beta=1-i \frac{2 \pi}{k} \frac{1}{2 N}+\mathcal{O}\left(\frac{1}{k^{2}}\right) \text { and } z=-i \frac{2 \pi}{k}+\mathcal{O}\left(\frac{1}{k^{2}}\right)
$$

and the knot diagrams are shown in Figure 3.1.
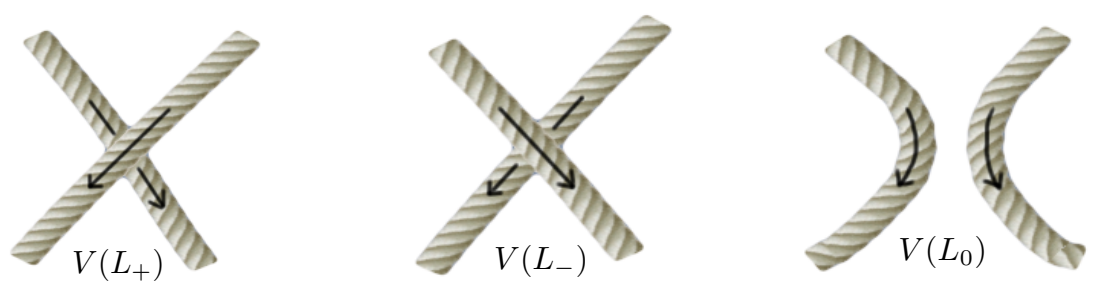

Figure 3.1: Knot diagrams

In the temporal gauge with complex coordinates and in representation $R, 3.29$ ) becomes

$$
W_{R}(C)=\operatorname{Tr}_{R} \mathcal{P} e^{-\oint_{c}\left(A_{z} d z+A_{\bar{z}} d \bar{z}\right)}
$$

Since $A_{z}$ is the derivative with respect to $A_{\bar{z}}$ and it acts on everything on its right, expanding this path ordered exponential leads to a very difficult calculation. To avoid this, instead of using the usual definition of the Wilson loop, we would like to use a Wilson loop-like observable defined as

$$
\mathcal{W}_{R}(C)=\operatorname{Tr}_{R} \mathcal{P} e^{-\oint_{c}\left(\mathcal{A}_{z} d z+A_{\bar{z}} d \bar{z}\right)}=\operatorname{Tr}_{R} U(x, x, C)
$$

where $\mathcal{A}_{z}$ is given by $\partial_{z} A_{\bar{z}}-\partial_{\bar{z}} \mathcal{A}_{z}+\left[\mathcal{A}_{z}, A_{\bar{z}}\right]=0$. Since $F_{z \bar{z}}=0$ from the Gauss' law, replacing $A_{z}$ with $\mathcal{A}_{z}$ does not change the general properties of the Wilson loop when evaluated on states that live on the constraint hyper-surface. Since $U$ is defined to be path independent, it 
would seem that the skein relations are trivially satisfied. However, this is not true since the path independence is only on $\Sigma$, because we are forcing flatness only on the $z \bar{z}$ component of the curvature. So, one is allowed to make small deformations in the time direction, piercing $\Sigma$ to get skein relations.

In the previous section, we have shown that the theory is given by the action $S_{W Z W}(H)$, thus we can use gauge invariant WZW currents $J_{\bar{z}}=-\partial_{\bar{z}} H H^{-1}$ and $J_{z}=H^{-1} \partial_{z} H$ to write gauge invariant observables similar to Wilson loops [22]. The gauge fields in $\mathcal{W}_{R}(C)$ can be written as $S L(N, \mathbb{C})$ transformed WZW currents;

$$
\begin{aligned}
& A_{\bar{z}}=-\partial_{\bar{z}} U U^{-1}=U^{\dagger-1} J_{\bar{z}} U^{\dagger}+U^{\dagger-1} \partial_{\bar{z}} U^{\dagger} \\
& \mathcal{A}_{z}=-\partial_{z} U U^{-1}=U^{\dagger-1} J_{z} U^{\dagger}+U^{\dagger-1} \partial_{z} U^{\dagger}
\end{aligned}
$$

Thus, we can write $\mathcal{W}$ in terms of $\mathrm{H}$

$$
\mathcal{W}_{R}(C, H)=\operatorname{Tr}_{R} \mathcal{P} e^{\oint{ }_{c}\left(\partial_{z} H H^{-1} d z+\partial_{\bar{z}} H H^{-1} d \bar{z}\right)}
$$

Since $\mathcal{W}$ commutes with the wave-functional, the expectation value of it can be written as

$$
\left\langle\mathcal{W}_{R}(C)\right\rangle=\int d \mu(H) e^{\left(2 c_{a}+k\right) S_{W Z W}(H)} \mathcal{W}_{R}(C, H)
$$




\section{CHAPTER 4 \\ TOPOLOGICALLY MASSIVE YANG-MILLS THEORY}

Topologically massive Yang-Mills action(TMYM) is given by

$$
\begin{aligned}
S_{T M Y M}= & S_{C S}+S_{Y M} \\
= & -\frac{k}{4 \pi} \int_{\Sigma \times\left[t_{i}, t_{f}\right]} d^{3} x \epsilon^{\mu \nu \alpha} \operatorname{Tr}\left(A_{\mu} \partial_{\nu} A_{\alpha}+\frac{2}{3} A_{\mu} A_{\nu} A_{\alpha}\right) \\
& -\frac{k}{4 \pi} \frac{1}{m} \int_{\Sigma \times\left[t_{i}, t_{f}\right]} d^{3} x \operatorname{Tr} F_{\mu \nu} F^{\mu \nu} .
\end{aligned}
$$

Here $m$ is called the topological mass (see section 2.5). Our definition of topological mass differs by a factor $\frac{k}{4 \pi}$ compared to the literature on this theory. We made this choice so that studying different values of $k$ does not change the balance of the theory in either pure Yang-Mills(YM) or pure Chern-Simons(CS) direction. That is decided only by the value of $m$. With this choice of constants, the equations of motion are $k$ free, as

$$
\epsilon^{\mu \alpha \beta} F_{\alpha \beta}+\frac{1}{m} D_{\nu} F^{\mu \nu}=0
$$

where $D_{\mu} \bullet=\partial_{\mu} \bullet+\left[A_{\mu}, \bullet\right]$. Following ref. [49], we simplify the notation with defining

$$
\tilde{A}_{\mu}=A_{\mu}+\frac{1}{2 m} \epsilon_{\mu \alpha \beta} F^{\alpha \beta}
$$

Here $\tilde{A}$ is not a field redefinition. It is just a shorthand notation to make equations easy to compare with pure CS theory. From 4.3 , it can be seen that $\tilde{A}_{\mu}$ transforms like a gauge field since $F^{\alpha \beta}$ is gauge covariant. For future convenience, using $\tilde{A}_{\mu}$ as a connection, we define a new covariant derivative $\tilde{D}_{\mu} \bullet=\partial_{\mu} \bullet+\left[\tilde{A}_{\mu}, \bullet\right]$. 
Using complex coordinates and choosing the temporal gauge, the conjugate momenta are given by $\tilde{A}$,

$$
\Pi^{a z}=\frac{i k}{4 \pi} \tilde{A}_{\bar{z}}^{a} \text { and } \Pi^{a \bar{z}}=-\frac{i k}{4 \pi} \tilde{A}_{z}^{a}
$$

with

$$
\tilde{A}_{z}=A_{z}+E_{z} \text { and } \tilde{A}_{\bar{z}}=A_{\bar{z}}+E_{\bar{z}}
$$

and where

$$
E_{z}=\frac{i}{2 m} F^{0 \bar{z}} \text { and } E_{\bar{z}}=-\frac{i}{2 m} F^{0 z}
$$

The conjugate momenta of TMYM theory transform like gauge fields and this feature gives the theory a Chern-Simons-like behavior in some sense. Thus, we will be able to borrow many of the features of CS theory in the following analysis.

The symplectic two-form for this theory is

$$
\begin{aligned}
\Omega & =\frac{i k}{4 \pi} \int_{\Sigma}\left(\delta \tilde{A}_{\bar{z}}^{a} \delta A_{z}^{a}+\delta A_{\bar{z}}^{a} \delta \tilde{A}_{z}^{a}\right) \\
& =\frac{i k}{4 \pi} \int_{\Sigma}\left(2 \delta A_{\bar{z}}^{a} \delta A_{z}^{a}+\delta E_{\bar{z}}^{a} \delta A_{z}^{a}+\delta A_{\bar{z}}^{a} \delta E_{z}^{a}\right) .
\end{aligned}
$$

From this equation, it can be seen that the TMYM phase space consists of two CS-like parts. This can be seen more clearly under a coordinate transformation. Instead of using $A_{z}$ and $A_{\bar{z}}$, we could use $B_{z}=\frac{1}{2}\left(A_{1}+i \tilde{A}_{2}\right), C_{z}=\frac{1}{2}\left(\tilde{A}_{1}+i A_{2}\right)$ and their complex conjugates. This would allow us to write $\Omega$ in the form $\int_{\Sigma}\left(\delta B_{z}^{a} \delta B_{\bar{z}}^{a}+\delta C_{z}^{a} \delta C_{\bar{z}}^{a}\right)$. Thus, the phase space of TMYM theory can be written as a sum of two CS phase spaces. 


\subsection{Gauss' Law}

In TMYM theory, infinitesimal gauge transformations are generated by the vector field

$$
\xi=-\int_{\Sigma}\left[\left(D_{z} \varepsilon\right)^{a} \frac{\delta}{\delta A_{z}^{a}}+\left(D_{\bar{z}} \varepsilon\right)^{a} \frac{\delta}{\delta A_{\bar{z}}^{a}}+\left(\tilde{D}_{z} \varepsilon\right)^{a} \frac{\delta}{\delta \tilde{A}_{z}^{a}}+\left(\tilde{D}_{\bar{z}} \varepsilon\right)^{a} \frac{\delta}{\delta \tilde{A}_{\bar{z}}^{a}}\right] .
$$

To find the Gauss' law, we look at the interior contraction

$$
\begin{aligned}
& i_{\xi} \Omega=-\frac{i k}{4 \pi} \int_{\Sigma} \varepsilon^{a}\left[-\left(D_{z} \delta \tilde{A}_{\bar{z}}\right)^{a}+\left(D_{\bar{z}} \delta \tilde{A}_{z}\right)^{a}-\left(\tilde{D}_{z} \delta A_{\bar{z}}\right)^{a}+\left(\tilde{D}_{\bar{z}} \delta A_{z}\right)^{a}\right] \\
& =-\frac{i k}{4 \pi} \int_{\Sigma} \varepsilon^{a}\left[-\left(D_{z} \delta A_{\bar{z}}\right)^{a}-\left(D_{z} \delta E_{\bar{z}}\right)^{a}+\left(D_{\bar{z}} \delta A_{z}\right)^{a}+\left(D_{\bar{z}} \delta E_{z}\right)^{a}\right. \\
& \left.-\left(D_{z} \delta A_{\bar{z}}\right)^{a}-\left[E_{z}, \delta A_{\bar{z}}\right]^{a}+\left(D_{\bar{z}} \delta A_{z}\right)^{a}+\left[E_{\bar{z}}, \delta A_{z}\right]^{a}\right] \\
& =-\delta\left(\frac{i k}{4 \pi} \int_{\Sigma} \varepsilon^{a}\left[2 F_{z \bar{z}}^{a}+\left(D_{z} E_{\bar{z}}\right)^{a}-\left(D_{\bar{z}} E_{z}\right)^{a}\right]\right) \text {. }
\end{aligned}
$$

Then using (2.140, we can write the Gauss' law operator for TMYM theory as

$$
G^{a}=\frac{i k}{4 \pi}\left[2 F_{z \bar{z}}^{a}+\left(D_{z} E_{\bar{z}}\right)^{a}-\left(D_{\bar{z}} E_{z}\right)^{a}\right]
$$

\subsection{The Wave-Functional}

We choose the Kähler polarization that makes $\psi$ only $A_{\bar{z}}$ and $\tilde{A}_{\bar{z}}$ dependent. The pre-quantum wave-functional is $\Phi\left[A_{z}, A_{\bar{z}}, \tilde{A}_{z}, \tilde{A}_{\bar{z}}\right]=e^{-\frac{1}{2} K} \psi\left[A_{\bar{z}}, \tilde{A}_{\bar{z}}\right]$, where $K$ is the Kähler potential given by $K=\frac{k}{4 \pi} \int_{\Sigma}\left(\tilde{A}_{\bar{z}}^{a} A_{z}^{a}+A_{\bar{z}}^{a} \tilde{A}_{z}^{a}\right)$. Now, we can write

$$
A_{z}^{a} \psi=\frac{4 \pi}{k} \frac{\delta}{\delta \tilde{A}_{\bar{z}}^{a}} \psi \text { and } \tilde{A}_{z}^{a} \psi=\frac{4 \pi}{k} \frac{\delta}{\delta A_{\bar{z}}^{a}} \psi
$$

We make an infinitesimal gauge transformation on $\psi$ as

$$
\delta_{\varepsilon} \psi\left[A_{\bar{z}}, \tilde{A}_{\bar{z}}\right]=\int d^{2} z\left(\delta_{\varepsilon} A_{\bar{z}}^{a} \frac{\delta \psi}{\delta A_{\bar{z}}^{a}}+\delta_{\varepsilon} \tilde{A}_{\bar{z}}^{a} \frac{\delta \psi}{\delta \tilde{A}_{\bar{z}}^{a}}\right) .
$$


Using 4.11) and $\delta A_{\bar{z}}^{a}=D_{\bar{z}} \varepsilon^{a}, \delta \tilde{A}_{\bar{z}}^{a}=\tilde{D}_{\bar{z}} \varepsilon^{a}$, we get

$$
\begin{aligned}
\delta_{\varepsilon} \psi & =\int d^{2} z \varepsilon^{a}\left(\tilde{D}_{\bar{z}} \frac{\delta}{\delta \tilde{A}_{\bar{z}}^{a}}+D_{\bar{z}} \frac{\delta}{\delta A_{\bar{z}}^{a}}\right) \psi \\
& =\frac{k}{4 \pi} \int d^{2} z \varepsilon^{a}\left(\partial_{z} \tilde{A}_{\bar{z}}^{a}+\partial_{z} A_{\bar{z}}^{a}-2 F_{z \bar{z}}-D_{z} E_{\bar{z}}+D_{\bar{z}} E_{z}\right) \psi
\end{aligned}
$$

The generator of infinitesimal gauge transformations for this theory is

$$
G^{a}=\frac{i k}{4 \pi}\left(2 F_{z \bar{z}}+D_{z} E_{\bar{z}}-D_{\bar{z}} E_{z}\right)
$$

while $G^{a}=\frac{i k}{2 \pi} F_{z \bar{z}}$ being the generator for the pure CS theory as $E$-fields go to zero $(m \rightarrow \infty)$. After applying the Gauss' law $G^{a} \psi=0, \delta_{\varepsilon} \psi$ becomes

$$
\delta_{\varepsilon} \psi=\frac{k}{4 \pi} \int d^{2} z \varepsilon^{a}\left(\partial_{\bar{z}} \tilde{A}_{z}^{a}+\partial_{\bar{z}} A_{z}^{a}\right) \psi .
$$

This equation is similar to 3.17 . As they transform identically, $\tilde{A}$ can be parametrized the same way as $A$, using a different $\operatorname{SL}(\mathrm{N}, \mathbb{C})$ matrix $\tilde{U}$,

$$
\tilde{A}_{\bar{z}}=-\partial_{\bar{z}} \tilde{U} \tilde{U}^{-1} \text { and } \tilde{A}_{z}=\tilde{U}^{\dagger-1} \partial_{z} \tilde{U}^{\dagger}
$$

The solution for the condition 4.15) is

$$
\psi\left[A_{\bar{z}}, \tilde{A}_{\bar{z}}\right]=\exp \left[\frac{k}{2}\left(S_{W Z W}(\tilde{U})+S_{W Z W}(U)\right)\right] \chi
$$

where $\chi$ is the gauge invariant part of $\psi$ that is required to satisfy the Schrödinger's equation. Notice that 4.17) reduces to Chern-Simons wave-functional (3.18) as expected, when topological mass approaches infinity, which is equivalent to dropping the tilde symbol. $\chi$ should be equal to one in this limit.

To understand the role of the new matrix $\tilde{U}$, we can relate it to $U$ by rewriting 4.5 as

$$
\partial_{\bar{z}} \tilde{U} \tilde{U}^{-1}=\partial_{\bar{z}} U U^{-1}+\frac{i}{2 m} F^{0 z}
$$


It turns out that $\tilde{U}$ is well behaved and solvable. Using the assumption $\tilde{U}=U M$, we can solve 4.18) for $M$, viz;

$$
M(z, \bar{z})=\mathcal{P} \exp \left(\frac{i}{2 m} \int_{0}^{\bar{z}} \mathcal{F}^{0 w} d \bar{w}\right)
$$

Here $\mathcal{F}^{0 z}=U^{-1} F^{0 z} U$ and it is gauge invariant. With this new gauge invariant matrix $M$, the electric field components can be written as

$$
E_{z}=U^{\dagger-1} M^{\dagger-1} \partial_{z} M^{\dagger} U^{\dagger} \text { and } E_{\bar{z}}=-U \partial_{\bar{z}} M M^{-1} U^{-1}
$$

\subsubsection{The Schrödinger's Equation}

In the temporal gauge, the Hamiltonian gets no contribution from the Chern-Simons

term. With no charges present, using $\alpha=\frac{4 \pi}{k}, B=\frac{i k}{2 \pi} F^{z \bar{z}}$ and Euclidean metric, the TMYM Hamiltonian is given by

$$
\mathcal{H}=\frac{m}{2 \alpha}\left(E_{\bar{z}}^{a} E_{z}^{a}+E_{z}^{a} E_{\bar{z}}^{a}\right)+\frac{\alpha}{m} B^{a} B^{a}
$$

Using (4.11) we get

$$
E_{z}^{a}(x) E_{\bar{z}}^{b}\left(x^{\prime}\right) \psi=\alpha\left(\frac{\delta}{\delta A_{\bar{z}}^{a}(x)}-\frac{\delta}{\delta \tilde{A}_{\bar{z}}^{a}(x)}\right)\left(\tilde{A}_{\bar{z}}^{b}\left(x^{\prime}\right)-A_{\bar{z}}^{b}\left(x^{\prime}\right)\right) \psi
$$

which gives the commutator

$$
\left[E_{z}^{a}(x), E_{\bar{z}}^{b}\left(x^{\prime}\right)\right]=-2 \alpha \delta^{a b} \delta^{(2)}\left(x-x^{\prime}\right)
$$

Here, $E_{z}^{a}$ can be interpreted as an annihilation operator and $E_{\bar{z}}^{b}$ as a creation operator $[14$. To get rid of the infinity, the Hamiltonian has to be normal ordered as

$$
\mathcal{H}=\frac{m}{\alpha} E_{\bar{z}}^{a} E_{z}^{a}+\frac{\alpha}{m} B^{a} B^{a} .
$$


To simplify the notation we write $\psi=\phi \chi$, where

$$
\phi=\exp \left[\frac{k}{2}\left(S_{W Z W}(\tilde{U})+S_{W Z W}(U)\right)\right]
$$

Derivatives of $\phi$ give the gauge field defined in (3.10) and its tilde version [33], as

$$
\tilde{A}_{z}^{a} \phi=\frac{4 \pi}{k} \frac{\delta \phi}{\delta A_{\bar{z}}^{a}}=\mathcal{A}_{z}^{a} \phi \text { and } A_{z}^{a} \phi=\frac{4 \pi}{k} \frac{\delta \phi}{\delta \tilde{A}_{\bar{z}}^{a}}=\tilde{\mathcal{A}}_{z}^{a} \phi
$$

The holomorphic component of the E-field acting on $\psi$ is

$$
E_{z}^{a} \psi=\frac{4 \pi}{k}\left(\frac{\delta \phi}{\delta A_{\bar{z}}^{a}}-\frac{\delta \phi}{\delta \tilde{A}_{\bar{z}}^{a}}\right) \chi+\frac{4 \pi}{k}\left(\frac{\delta \chi}{\delta A_{\bar{z}}^{a}}-\frac{\delta \chi}{\delta \tilde{A}_{\bar{z}}^{a}}\right) \phi
$$

With defining $\mathcal{E}_{z}=\tilde{\mathcal{A}}_{z}-\mathcal{A}_{z}=-U \partial_{z} M M^{-1} U^{-1}$, we can write

$$
E_{z}^{a} \psi=-\mathcal{E}_{z}^{a} \psi+\frac{4 \pi}{k}\left(\frac{\delta \chi}{\delta A_{\bar{z}}^{a}}-\frac{\delta \chi}{\delta \tilde{A}_{\bar{z}}^{a}}\right) \phi
$$

The magnetic field acting on $\psi$ is

$$
\begin{aligned}
F_{z \bar{z}}^{a} \psi & =\left(\partial_{z} A_{\bar{z}}^{a}-D_{\bar{z}} A_{z}^{a}\right) \psi \\
& =D_{\bar{z}}\left(\mathcal{A}_{z}^{a}-A_{z}^{a}\right) \psi \\
& =D_{\bar{z}}\left(-\mathcal{E}_{z}^{a} \psi-\frac{4 \pi}{k} \frac{\delta \chi}{\delta \tilde{A}_{\bar{z}}^{a}} \phi\right)
\end{aligned}
$$

The vacuum wave-functional is given by $\mathcal{H} \psi_{0}=0$, or

$$
E_{\bar{z}}^{a} E_{z}^{a} \psi_{0}+\frac{1}{64 m^{2}} F_{z \bar{z}}^{a} F_{z \bar{z}}^{a} \psi_{0}=0
$$

The first term in (5.24) is second order in $1 / m$, while the Gauss' law forces the second term to be fourth order. We will continue our analysis with finite large values of $m$ where the potential energy term is negligible. Then, $E_{z}^{a}$ annihilates the vacuum as

$$
E_{z}^{a} \psi_{0}=0
$$


This condition is solved by writing

$$
\chi_{0}=\exp \left(-\frac{k}{8 \pi} \int_{\Sigma}\left(\tilde{A}_{\bar{z}}^{a}-A_{\bar{z}}^{a}\right) \mathcal{E}_{z}^{a}\right)=\exp \left(-\frac{k}{8 \pi} \int_{\Sigma} E_{\bar{z}}^{a} \mathcal{E}_{z}^{a}\right) .
$$

This solution is gauge invariant as required. In terms of $S L(N, \mathbb{C})$ matrices, it can be written as a function of only the gauge invariant matrix $M$, which was defined in 4.19,

$$
\chi_{0}=\exp \left(\frac{k}{4 \pi} \int_{\Sigma} \operatorname{Tr}\left(E_{\bar{z}} \mathcal{E}_{z}\right)\right)=\exp \left(-\frac{k}{4 \pi} \int_{\Sigma} \operatorname{Tr}\left(\partial_{\bar{z}} M M^{-1} \partial_{z} M M^{-1}\right)\right) .
$$

\subsection{The Measure}

Using the symplectic two-form (4.7) we write the metric

$$
\begin{aligned}
d s_{\mathfrak{A}}^{2} & =-4 \int \operatorname{Tr}\left(\delta \tilde{A}_{\bar{z}} \delta A_{z}+\delta A_{\bar{z}} \delta \tilde{A}_{z}\right) \\
& =4 \int \operatorname{Tr}\left[\tilde{D}_{\bar{z}}\left(\delta \tilde{U} \tilde{U}^{-1}\right) D_{z}\left(U^{\dagger-1} \delta U^{\dagger}\right)+D_{\bar{z}}\left(\delta U U^{-1}\right) \tilde{D}_{z}\left(\tilde{U}^{\dagger-1} \delta \tilde{U}^{\dagger}\right)\right]
\end{aligned}
$$

Similar to the analysis in 3.3 , we find that the gauge invariant measure for this case to be

$$
d \mu(\mathfrak{A})=\operatorname{det}\left(\tilde{D}_{\bar{z}} D_{z}\right) \operatorname{det}\left(D_{\bar{z}} \tilde{D}_{z}\right) d \mu\left(\tilde{U}^{\dagger} U\right) d \mu\left(U^{\dagger} \tilde{U}\right)
$$

where for a certain choice of local counter terms $\left(\int \operatorname{Tr}\left(\tilde{A}_{\bar{z}} A_{z}+\tilde{A}_{z} A_{\bar{z}}\right)\right)$,

$$
\operatorname{det}\left(\tilde{D}_{\bar{z}} D_{z}\right) \operatorname{det}\left(D_{\bar{z}} \tilde{D}_{z}\right)=\text { constant } \times e^{2 c_{A}\left(S_{W Z W}\left(\tilde{U}^{\dagger} U\right)+S_{W Z W}\left(U^{\dagger} \tilde{U}\right)\right)} .
$$

To simplify the notation we define a new matrix $N=\tilde{U}^{\dagger} U$. Since $\tilde{U}$ transforms like $U, N$ is gauge invariant. With this definition the measure becomes

$$
d \mu(\mathfrak{A})=\text { constant } \times e^{2 c_{A}\left(S_{W Z W}(N)+S_{W Z W}\left(N^{\dagger}\right)\right)} d \mu(N) d \mu\left(N^{\dagger}\right) .
$$


To find the inner product, using the Polyakov-Wiegmann identity we write

$$
e^{-K_{T M Y M}} \psi_{T M Y M}^{*} \psi_{T M Y M}=e^{\frac{k}{2}\left(S_{W Z W}(N)+S_{W Z W}\left(N^{\dagger}\right)\right)} \chi_{0}^{*} \chi_{0}
$$

and from 4.32$) \chi_{0}^{*} \chi_{0}$ (for large $m$ ) is

$$
\chi_{0}^{*} \chi_{0}=\exp \left(-\frac{k}{8 \pi} \int_{\Sigma}\left(E_{z}^{a} \mathcal{E}_{\bar{z}}^{a}+E_{\bar{z}}^{a} \mathcal{E}_{z}^{a}\right)\right) .
$$

Then the inner product for the vacuum state is

$$
\left\langle\psi_{0} \mid \psi_{0}\right\rangle=\int d \mu(N) d \mu\left(N^{\dagger}\right) e^{\left(2 c_{A}+\frac{k}{2}\right)\left(S_{W Z W}(N)+S_{W Z W}\left(N^{\dagger}\right)\right)} e^{-\frac{k}{8 \pi} \int\left(E_{z}^{a} \mathcal{E}_{\bar{z}}^{a}+E_{\bar{z}}^{a} \mathcal{E}_{z}^{a}\right)} .
$$

Since $\chi_{0}^{*} \chi_{0}=1+\mathcal{O}\left(1 / m^{2}\right)$, we can neglect the second and higher order contributions at large scales compared to $1 / m$, which leads to an almost topological theory in the near CS limit as

$$
\left\langle\psi_{0} \mid \psi_{0}\right\rangle_{T M Y M_{k}} \approx \int d \mu(N) d \mu\left(N^{\dagger}\right) e^{\left(2 c_{A}+\frac{k}{2}\right)\left(S_{W Z W}(N)+S_{W Z W}\left(N^{\dagger}\right)\right)}=\langle\psi \mid \psi\rangle_{C S_{k / 2}}\langle\psi \mid \psi\rangle_{C S_{k / 2}} .
$$

Here the label $T M Y M_{k}$ means that the inner product is calculated for TMYM theory with CS level number $k$. Similarly, $C S_{k / 2}$ means the inner product is calculated for pure CS theory with level $k / 2$. On the pure CS side, it is important to make this replacement of the level number to make the equivalence work. These two half-CS theories are not separately gauge invariant for odd values of $k$, but the sum of the two is. Each piece transforms as $\frac{1}{2} S_{C S}\left(A^{g}\right) \rightarrow \frac{1}{2} S_{C S}(A)+\pi k \omega(g)$ where $\omega(g)$ is the winding number. Then, the sum of the two will bring an extra $2 \pi k \omega(g)$ that will not change the value of the path integral, even for odd values of $k$. In other words, any integer value of $k$ is sufficient to make the left hand side of 4.41 gauge invariant. But if one wants to make the two split CS parts separately gauge invariant on the right hand side of 4.41, even values of $k$ must be chosen. 
This equivalence at large distances $(d>1 / m)$ comes from the fact that the phase space of TMYM theory is a direct sum of two CS-like phase spaces. Thus, the classical equivalence discussed in refs. 18, 19,29 does not work at the quantum level as it is, because of different phase space dimensionality of two theories.

In (5.31), gauge invariance can be obtained in a different way by choosing other counter terms. Choosing $\int \operatorname{Tr}\left(A_{\bar{z}} A_{z}+\tilde{A}_{z} \tilde{A}_{\bar{z}}\right)$ leads to

$$
d \mu(\mathfrak{A})=\text { constant } \times e^{2 c_{A}\left(S_{W Z W}(H)+S_{W Z W}(\tilde{H})\right)} d \mu(H) d \mu(\tilde{H})
$$

With this option $e^{-K} \psi^{*} \psi$ part differs by $e^{-\int E^{2}}=1+\mathcal{O}\left(1 / m^{2}\right)$ and the inner product can still be written in the form

$$
\left\langle\psi_{0} \mid \psi_{0}\right\rangle_{T M Y M_{k}} \approx \int d \mu(H) d \mu(\tilde{H}) e^{\left(2 c_{A}+\frac{k}{2}\right)\left(S_{W Z W}(H)+S_{W Z W}(\tilde{H})\right)}=\langle\psi \mid \psi\rangle_{C S_{k / 2}}\langle\psi \mid \psi\rangle_{C S_{k / 2}}
$$

Thus, the Chern-Simons splitting can still be observed in the near Chern-Simons limit. Just like $N$ and $N^{\dagger}, H$ and $\tilde{H}$ are elements of $S L(N, \mathbb{C}) / S U(N)$. However, the $N, N^{\dagger}$ seems to be a more natural choice than $H, \tilde{H}$ because tilde and non-tilde variables are mixed in (4.7).

As we have seen in section 2.7, the gravitational analogue of TMYM theory has a very similar CS splitting in the near CS limit, as

$$
S[e] \approx \frac{1}{2 \mu} S_{C S}\left[A^{+}[e]\right]+\frac{1}{2 \mu} S_{C S}\left[A^{-}[e]\right]
$$

This is analogous to what we have observed for TMYM theory at large distances, as we have predicted in section 2.7 . 


\subsection{Wilson Loops in Topologically Massive Yang-Mills Theory}

With the new gauge field $\tilde{A}$, we can define a new loop operator

$$
T_{R}(C)=\operatorname{Tr}_{R} \mathcal{P} e^{-\oint_{c} \tilde{A}_{\mu} d x^{\mu}}
$$

Just as the traditional Wilson loop, this operator is gauge invariant and it is an observable of the theory. To make a physical interpretation of this loop, we will check to see if it satisfies a 't Hooft-like algebra with the Wilson loop. To simplify the calculation, we will look at the abelian case with the following loops that live on $\Sigma$

$$
W(C)=e^{i \oint_{c}\left(A_{z} d z+A_{\bar{z}} d \bar{z}\right)} \text { and } T(C)=e^{i \oint_{c}\left(\tilde{A}_{z} d z+\tilde{A}_{\bar{z}} d \bar{z}\right)}
$$

For the abelian case, the canonical relations differ from 4.11 by a factor of 2 . Then two loop operators satisfy a ’t Hooft-like algebra

$$
T\left(C_{1}\right) W\left(C_{2}\right)=e^{\frac{2 \pi i}{k} l\left(C_{1}, C_{2}\right)} W\left(C_{2}\right) T\left(C_{1}\right)
$$

where $l\left(C_{1}, C_{2}\right)$ is the intersection number of $C_{1}$ and $C_{2}$, which can only take values $0, \pm 1$. We cannot get a Dirac-like quantization condition since $k$ appears in the denominator and we want it to be a large integer to make the skein relations work. Therefore, the only option to make these operators commute is to have $l\left(C_{1}, C_{2}\right)=0$ thus, two loops cannot share a point. Equation (4.47) lets us to interpret $T(C)$ as a 't Hooft-like loop for TMYM theory.

Working with the holomorphic polarization leads to the same problem we had in the pure Chern-Simons case. $A_{z}$ and $\tilde{A}_{z}$ are derivatives with respect to $\tilde{A}_{\bar{z}}$ and $A_{\bar{z}}$. This makes the path ordered exponential very complicated. To avoid this problem, we use a similar technique: Instead of using the traditional Wilson loop, we will calculate the expectation 
value of two loop operators that we define by $\operatorname{Tr} U(x, x, C)$ and $\operatorname{Tr} \tilde{U}(x, x, C)$ or

$$
\mathcal{W}_{R}(C)=\operatorname{Tr}_{R} \mathcal{P} e^{-\oint_{c}\left(\mathcal{A}_{z} d z+A_{\bar{z}} d \bar{z}\right)} \text { and } \mathcal{T}_{R}(C)=\operatorname{Tr}_{R} \mathcal{P} e^{-\oint_{c}\left(\tilde{\mathcal{A}}_{z} d z+\tilde{A}_{\bar{z}} d \bar{z}\right)}
$$

Once again these can be written in terms of WZW currents $-\partial_{\bar{z}} N N^{-1},-\partial_{z} N N^{-1},-\partial_{\bar{z}} N^{\dagger} N^{\dagger-1}$ and $-\partial_{z} N^{\dagger} N^{\dagger-1}$ as

$$
\mathcal{W}_{R}(C)=\operatorname{Tr}_{R} \mathcal{P} e^{\oint_{c}\left(\partial_{z} N N^{-1} d z+\partial_{\bar{z}} N N^{-1} d \bar{z}\right)} \text { and } \mathcal{T}_{R}(C)=\operatorname{Tr}_{R} \mathcal{P} e^{\oint_{c}\left(\partial_{z} N^{\dagger} N^{\dagger-1} d z+\partial_{\bar{z}} N^{\dagger} N^{\dagger-1} d \bar{z}\right)}
$$

These WZW currents are $S L(N, \mathbb{C})$ transformed $A$ and $\tilde{A}$ fields, just as in 3.34 .

There is an interesting expectation value that we can calculate;

$$
\begin{aligned}
\left\langle\mathcal{W}_{R_{1}}\left(C_{1}\right) \mathcal{T}_{R_{2}}\left(C_{2}\right)\right\rangle= & \int d \mu(\mathscr{A}) \psi_{0}^{*} \mathcal{W}_{R_{1}}\left(C_{1}\right) \mathcal{T}_{R_{2}}\left(C_{2}\right) \psi_{0} \\
= & \int d \mu(N) d \mu\left(N^{\dagger}\right) e^{\left(2 c_{A}+\frac{k}{2}\right)\left(S_{W Z W}(N)+S_{W Z W}\left(N^{\dagger}\right)\right)} \mathcal{W}_{R_{1}}\left(C_{1}, N\right) \mathcal{T}_{R_{2}}\left(C_{2}, N^{\dagger}\right) \\
& +\mathcal{O}\left(1 / m^{2}\right) .
\end{aligned}
$$

This leads to an equivalence between the observables of CS and TMYM theory in the near CS limit. $\mathcal{W}_{R}(C)$ being only $N$ dependent and $\mathcal{T}_{R}(C)$ being only $N^{\dagger}$ dependent lets us to write

$$
\begin{aligned}
\left\langle\mathcal{W}_{R}(C)\right\rangle_{T M Y M_{2 k}} & =\left\langle\mathcal{W}_{R}(C)\right\rangle_{C S_{k}}+\mathcal{O}\left(1 / m^{2}\right), \\
\left\langle\mathcal{T}_{R}(C)\right\rangle_{T M Y M_{2 k}} & =\left\langle\mathcal{W}_{R}(C)\right\rangle_{C S_{k}}+\mathcal{O}\left(1 / m^{2}\right)
\end{aligned}
$$

and

$$
\left\langle\mathcal{W}_{R_{1}}\left(C_{1}\right) \mathcal{T}_{R_{2}}\left(C_{2}\right)\right\rangle_{T M Y M_{2 k}}=\left(\left\langle\mathcal{W}_{R_{1}}\left(C_{1}\right)\right\rangle_{C S_{k}}\right)\left(\left\langle\mathcal{W}_{R_{2}}\left(C_{2}\right)\right\rangle_{C S_{k}}\right)+\mathcal{O}\left(1 / m^{2}\right)
$$


To generalize these for $n$ loops, we can write

$$
\begin{aligned}
\left\langle\mathcal{W}_{R_{1}}\left(C_{1}\right) \ldots \mathcal{W}_{R_{n}}\left(C_{n}\right)\right\rangle_{T M Y M_{2 k}} & \left.=\left\langle\mathcal{W}_{R_{1}}\left(C_{1}\right) \ldots \mathcal{W}_{R_{n}}\left(C_{n}\right)\right)\right\rangle_{C S_{k}}+\mathcal{O}\left(1 / m^{2}\right), \\
\left\langle\mathcal{T}_{R_{1}}\left(C_{1}\right) \ldots \mathcal{T}_{R_{n}}\left(C_{n}\right)\right\rangle_{T M Y M_{2 k}} & \left.=\left\langle\mathcal{W}_{R_{1}}\left(C_{1}\right) \ldots \mathcal{W}_{R_{n}}\left(C_{n}\right)\right)\right\rangle_{C S_{k}}+\mathcal{O}\left(1 / m^{2}\right)
\end{aligned}
$$

and for mixed $n$ Wilson-like and $m$ 't Hooft-like loops,

$$
\begin{aligned}
\left\langle\mathcal{W}_{R_{1}}\left(C_{1}\right) \ldots\right. & \left.\mathcal{W}_{R_{n}}\left(C_{n}\right) \mathcal{T}_{R_{1}^{\prime}}\left(C_{1}^{\prime}\right) \ldots \mathcal{T}_{R_{m}^{\prime}}\left(C_{m}^{\prime}\right)\right\rangle_{T M Y M_{2 k}} \\
& =\left(\left\langle\mathcal{W}_{R_{1}}\left(C_{1}\right) \ldots \mathcal{W}_{R_{n}}\left(C_{n}\right)\right\rangle_{C S_{k}}\right)\left(\left\langle\mathcal{W}_{R_{1}^{\prime}}\left(C_{1}^{\prime}\right) \ldots \mathcal{W}_{R_{m}^{\prime}}\left(C_{m}^{\prime}\right)\right\rangle_{C S_{k}}\right) \\
& +\mathcal{O}\left(1 / m^{2}\right) .
\end{aligned}
$$

Although (4.41) is gauge invariant even for odd values of $k$ as we have explained before, writing WLEVs of TMYM theory in terms of WLEVs of CS theory requires the two split CS parts to be separately gauge invariant. For this reason, we have used even level numbers on the left hand side of 4.51) and 4.52. Notice that this gauge invariance issue arose only because we wanted to arrive to an equivalence between the observables of TMYM and CS theories. Otherwise, $\left\langle\mathcal{W}_{R_{1}}\left(C_{1}\right) \mathcal{T}_{R_{2}}\left(C_{2}\right)\right\rangle_{T M Y M_{k}}$ is gauge invariant in its own right for all integer values of $k$.

It seems like this set of equivalences also work for the case where $\Sigma=S^{1} \times S^{1}$. On a torus, similar to 3.28, TMYM wave-functional becomes

$$
\psi\left[A_{\bar{z}}, \tilde{A}_{\bar{z}}\right]=\exp \left[\frac{k}{2}\left(S_{W Z W}(\tilde{V})+S_{W Z W}(V)\right)\right] \Upsilon(\tilde{a}) \Upsilon(a) \chi .
$$

On the TMYM side, one needs to integrate over $V, \tilde{V}, a, \tilde{a}$ and on the Chern-Simons side only over $V$ and $a$. Although it requires a more careful analysis, 4.52 seem to work on a torus as well. In principle, there is no reason to expect it to not work on any orientable $\Sigma$. 


\section{CHAPTER 5 \\ 2+1D PURE YANG-MILLS THEORY AT LARGE DISTANCES}

In chapter 4, we have seen that the inner product of topologically massive YangMills(TMYM) theory can be written as

$$
\left\langle\psi_{0} \mid \psi_{0}\right\rangle_{T M Y M_{k}}=\langle\psi \mid \psi\rangle_{C S_{k / 2}}\langle\psi \mid \psi\rangle_{C S_{k / 2}}+\mathcal{O}\left(1 / m^{2}\right)
$$

which is analogous to the Chern-Simons(CS) splitting of topologically massive AdS gravity model, which can be seen in 2.29). In the pure Einstein-Hilbert $\operatorname{limit}(\mu \rightarrow \infty)$, 2.27) becomes

$$
S[e]=\frac{1}{2} S_{C S}\left[A^{-}[e]\right]-\frac{1}{2} S_{C S}\left[A^{+}[e]\right]
$$

As we have seen in section 2.7, the corresponding gauge theory for this limit is the pure Yang-Mills(YM) theory. With this motivation, we will study the pure YM theory in this chapter, following ref. 50. We will look at large distances to see whether or not the analogy can be extended to this limit and a similar splitting can be observed.

\section{$5.1 \quad$ Yang-Mills Theory In 2+1 Dimensions}

The YM action is given by

$$
S_{Y M}=-\frac{k}{4 \pi} \frac{1}{4 m} \int_{\Sigma \times\left[t_{i}, t_{f}\right]} d^{3} x \operatorname{Tr}\left(F_{\mu \nu} F^{\mu \nu}\right) .
$$

The constant $\frac{k}{4 \pi}$ is normally not necessary but here it is inserted for future convenience. The equations of motion are given by $D_{\nu} F^{\mu \nu}=0$. 
In the temporal gauge $A_{0}=0$ with complex coordinates, the conjugate momenta are given by

$$
\Pi^{a z}=\frac{k}{8 \pi m} F^{a 0 z} \text { and } \Pi^{a \bar{z}}=\frac{k}{8 \pi m} F^{a 0 \bar{z}}
$$

With $E_{z}=\frac{i}{2 m} F^{0 \bar{z}}$ and $E_{\bar{z}}=-\frac{i}{2 m} F^{0 z}$, the momenta can be rewritten as

$$
\Pi^{a z}=\frac{i k}{4 \pi} E_{\bar{z}}^{a} \text { and } \Pi^{a \bar{z}}=-\frac{i k}{4 \pi} E_{z}^{a}
$$

With these coordinates, the symplectic two form is given by

$$
\Omega=\frac{i k}{4 \pi} \int_{\Sigma}\left(\delta E_{\bar{z}}^{a} \delta A_{z}^{a}+\delta A_{\bar{z}}^{a} \delta E_{z}^{a}\right) .
$$

Similar to the calculation in section 3.1 and section 4.1, it can be shown that the generator of infinitesimal gauge transformations is $G^{a}=\frac{i k}{4 \pi}\left(D_{z} E_{\bar{z}}-D_{\bar{z}} E_{z}\right)^{a}$.

To show how CS splitting happens, we need to write $\Omega$ in two $C S$-like parts. To do this, we will use the coordinates

$$
\tilde{A}_{i}=A_{i}+E_{i} \text { and } \hat{A}_{i}=A_{i}-E_{i}
$$

Since $E_{i}$ is gauge covariant, both $\tilde{A}_{i}$ and $\hat{A}_{i}$ transform like gauge fields. Now, $\Omega$ can be written as

$$
\Omega=\frac{i k}{4 \pi} \int_{\Sigma}\left(\delta \tilde{A}_{\bar{z}}^{a} \delta A_{z}^{a}-\delta A_{\bar{z}}^{a} \delta \hat{A}_{z}^{a}\right) .
$$

The phase space is Kähler with the potential

$$
K=\frac{k}{4 \pi} \int_{\Sigma}\left(\tilde{A}_{\bar{z}}^{a} A_{z}^{a}-A_{\bar{z}}^{a} \hat{A}_{z}^{a}\right) .
$$


Similar to what we have done before, we will parametrize the gauge fields. Since $\tilde{A}_{i}$ and $\hat{A}_{i}$ transform like gauge fields, they can be parametrized like one. Using new $S L(N, \mathbb{C})$ matrices $\tilde{U}$ and $\hat{U}$, defined similar to 3.9$)$, we can write

$$
\tilde{A}_{\bar{z}}=-\partial_{\bar{z}} \tilde{U} \tilde{U}^{-1} \text { and } \tilde{A}_{z}=\tilde{U}^{\dagger-1} \partial_{z} \tilde{U}^{\dagger}
$$

and

$$
\hat{A}_{\bar{z}}=-\partial_{\bar{z}} \hat{U} \hat{U}^{-1} \text { and } \hat{A}_{z}=\hat{U}^{\dagger-1} \partial_{z} \hat{U}^{\dagger}
$$

Also, $\tilde{\mathcal{A}}$ and $\hat{\mathcal{A}}$ can be written similar to 3.10$)$. We can use $(5.7)$ to relate the matrices $\tilde{U}$ and $\hat{U}$ with $U$. Assuming $\tilde{U}=U M$ and $\hat{U}=U N$, we can solve 5.7 with

$$
M(z, \bar{z})=N^{-1}(z, \bar{z})=\mathcal{P} \exp \left(\frac{i}{2 m} \int_{0}^{\bar{z}} \mathcal{F}^{0 w} d \bar{w}\right)
$$

where $\mathcal{F}^{0 z}=U^{-1} F^{0 z} U$. It can be seen that $\mathcal{F}$ is gauge invariant, so is $M$. With these new matrices, we can write the $E$-field components as

$$
\begin{aligned}
& E_{z}=U^{\dagger-1} M^{\dagger-1} \partial_{z} M^{\dagger} U^{\dagger}=-U^{\dagger-1} N^{\dagger-1} \partial_{z} N^{\dagger} U^{\dagger} \\
& E_{\bar{z}}=-U \partial_{\bar{z}} M M^{-1} U^{-1}=U \partial_{\bar{z}} N N^{-1} U^{-1}
\end{aligned}
$$

We can also write the $\mathcal{E}$-fields that are given by $\mathcal{E}_{i}=\tilde{\mathcal{A}}_{i}-\mathcal{A}_{i}=\mathcal{A}_{i}-\hat{\mathcal{A}}_{i}$, as

$$
\begin{aligned}
& \mathcal{E}_{z}=-U \partial_{z} M M^{-1} U^{-1}=U \partial_{z} N N^{-1} U^{-1} \\
& \mathcal{E}_{\bar{z}}=U^{\dagger-1} M^{\dagger-1} \partial_{\bar{z}} M^{\dagger} U^{\dagger}=-U^{\dagger-1} N^{\dagger-1} \partial_{\bar{z}} N^{\dagger} U^{\dagger} .
\end{aligned}
$$

\subsection{The Wave-Functional}

Choosing the holomorphic polarization gives $\Phi\left[A_{z}, A_{\bar{z}}, \hat{A}_{z}, \tilde{A}_{\bar{z}}\right]=e^{-\frac{1}{2} K} \psi\left[A_{\bar{z}}, \tilde{A}_{\bar{z}}\right]$, where $K$ is the Kähler potential given in 5.9$), \Phi$ and $\psi$ are the pre-quantum and quantum wave- 
functionals. $\chi$ is a function of both $A_{\bar{z}}$ and $\tilde{A}_{\bar{z}}$ and since it is gauge invariant, it has to be a function of the difference of these variables, which is $E_{\bar{z}}$.

From (5.8), upon quantization we can write

$$
A_{z}^{a} \psi=\frac{4 \pi}{k} \frac{\delta \psi}{\delta \tilde{A}_{\bar{z}}^{a}} \text { and } \hat{A}_{z}^{a} \psi=-\frac{4 \pi}{k} \frac{\delta \psi}{\delta A_{\bar{z}}^{a}}
$$

Now, we make an infinitesimal gauge transformation on $\psi$,

$$
\delta_{\epsilon} \psi\left[A_{\bar{z}}, \tilde{A}_{\bar{z}}\right]=\int d^{2} z\left(\delta_{\epsilon} A_{\bar{z}}^{a} \frac{\delta \psi}{\delta A_{\bar{z}}^{a}}+\delta_{\epsilon} \tilde{A}_{\bar{z}}^{a} \frac{\delta \psi}{\delta \tilde{A}_{\bar{z}}^{a}}\right)
$$

Using (5.15), $\delta A_{\bar{z}}^{a}=D_{\bar{z}} \epsilon^{a}$ and $\delta \tilde{A}_{\bar{z}}^{a}=\tilde{D}_{\bar{z}} \epsilon^{a}, 5.16$ can be rewritten as

$$
\begin{aligned}
\delta_{\epsilon} \psi & =\int d^{2} z \epsilon^{a}\left(D_{\bar{z}} \frac{\delta}{\delta A_{\bar{z}}^{a}}+\tilde{D}_{\bar{z}} \frac{\delta}{\delta \tilde{A}_{\bar{z}}^{a}}\right) \psi \\
& =\frac{k}{4 \pi} \int d^{2} z \epsilon^{a}\left(\tilde{D}_{\bar{z}} A_{z}^{a}-D_{\bar{z}} \hat{A}_{z}^{a}\right) \psi .
\end{aligned}
$$

Using (5.7), (5.17) becomes

$$
\delta_{\epsilon} \psi=\frac{k}{4 \pi} \int d^{2} z \epsilon^{a}\left(\partial_{z} E_{\bar{z}}^{a}-D_{z} E_{\bar{z}}^{a}+D_{\bar{z}} E_{z}^{a}\right) \psi
$$

After applying the Gauss' law $G^{a} \psi=0$, we get

$$
\begin{aligned}
\delta_{\epsilon} \psi & =\frac{k}{4 \pi} \int d^{2} z \epsilon^{a}\left(\partial_{z} E_{\bar{z}}^{a}\right) \psi \\
& =\frac{k}{4 \pi} \int d^{2} z \epsilon^{a}\left(\partial_{z} \tilde{A}_{\bar{z}}^{a}-\partial_{z} A_{\bar{z}}^{a}\right) \psi \\
& =\frac{k}{4 \pi} \int d^{2} z \epsilon^{a}\left(\partial_{z} A_{\bar{z}}^{a}-\partial_{z} \hat{A}_{\bar{z}}^{a}\right) \psi
\end{aligned}
$$

This condition is solved by $\psi=\phi \chi$ with

$$
\phi\left[A_{\bar{z}}, \tilde{A}_{\bar{z}}\right]=\exp \left[\frac{k}{2}\left(S_{W Z W}(\tilde{U})-S_{W Z W}(U)\right)\right]
$$


or equally

$$
\phi\left[A_{\bar{z}}, \tilde{A}_{\bar{z}}\right]=\exp \left[\frac{k}{2}\left(S_{W Z W}(U)-S_{W Z W}(\hat{U})\right)\right] .
$$

The equivalence of (5.20) and (5.21) can be shown by using the Polyakov-Wiegmann(PW) identity with $\tilde{U}=U M$ and $\hat{U}=U M^{-1}$.

Holomorphic components of gauge fields acting on $\phi$ gives the $\mathcal{A}$ and $\tilde{\mathcal{A}}$ fields, as

$$
A_{z}^{a} \phi=\frac{4 \pi}{k} \frac{\delta \phi}{\delta \tilde{A}_{\bar{z}}^{a}}=\mathcal{A}_{z}^{a} \phi \text { and } \hat{A}_{z}^{a} \phi=-\frac{4 \pi}{k} \frac{\delta \phi}{\delta A_{\bar{z}}^{a}}=\tilde{\mathcal{A}}_{z}^{a} \phi
$$

\subsubsection{The Schrödinger's Equation}

The YM Hamiltonian is given by

$$
\mathcal{H}=T+V \text { with } T=\frac{m}{\alpha} E_{\bar{z}}^{a} E_{z}^{a} \text { and } V=\frac{\alpha}{m} B^{a} B^{a}
$$

where $\alpha=\frac{4 \pi}{k}, B=\frac{i k}{2 \pi} F^{z \bar{z}}$. We are interested in finding the vacuum wave-functional, which is given by $\mathcal{H} \psi_{0}=0$, or with using Euclidean metric,

$$
E_{\bar{z}}^{a} E_{z}^{a} \psi_{0}+\frac{1}{64 m^{2}} F_{z \bar{z}}^{a} F_{z \bar{z}}^{a} \psi_{0}=0
$$

For both YM and TMYM theories, $\chi$ is typically in the form of $e^{-\frac{1}{m^{2}} \int F^{2}} 15,22,49$, (except for very small distances), since they have the same Hamiltonian in the temporal gauge. This exponential decay behavior cannot be polarization dependent and it comes from the existence of a mass gap. Thus, we expect it to be valid here as well. To check this assumption, we will first focus on the potential energy term. Using (5.22), the B-field acting on $\psi$ is 


$$
\begin{aligned}
F_{z \bar{z}}^{a} \psi & =\left(\partial_{z} A_{\bar{z}}^{a}-D_{\bar{z}} A_{z}^{a}\right) \psi \\
& =D_{\bar{z}}\left(\mathcal{A}_{z}^{a}-A_{z}^{a}\right) \psi \\
& =D_{\bar{z}}\left(-\mathcal{E}_{z}^{a} \psi-\frac{4 \pi}{k} \frac{\delta \chi}{\delta \tilde{A}_{\bar{z}}^{a}} \phi\right),
\end{aligned}
$$

where $\mathcal{E}_{z}$ is given by $\tilde{\mathcal{A}}_{z}-\mathcal{A}_{z}$. If we consider only the potential energy term, the gauge invariant part of the vacuum wave-function is given by

$$
\chi=\exp \left(-\frac{k}{4 \pi} \int_{\Sigma} E_{\bar{z}}^{a} \mathcal{E}_{z}^{a}\right)=\exp \left(-\frac{k}{4 \pi} \int_{\Sigma}\left(\tilde{A}_{\bar{z}}^{a}-A_{\bar{z}}^{a}\right) \mathcal{E}_{z}^{a}\right)=1+\mathcal{O}\left(1 / m^{2}\right) .
$$

Now, to study the kinetic energy term we look at $E_{z} \psi$. Using (5.22), we can write

$$
\begin{aligned}
E_{z}^{a} \psi & =\left(A_{z}^{a}-\hat{A}_{z}^{a}\right) \phi \chi \\
& =\mathcal{E}_{z}^{a} \psi+\frac{4 \pi}{k} \phi\left(\frac{\delta \chi}{\delta \tilde{A}_{\bar{z}}^{a}}+\frac{\delta \chi}{\delta A_{\bar{z}}^{a}}\right) .
\end{aligned}
$$

Since $\chi$ is gauge invariant, we can write $\chi=\chi\left[\tilde{A}_{\bar{z}}^{a}, A_{\bar{z}}^{a}\right]=\chi\left[\tilde{A}_{\bar{z}}^{a}-A_{\bar{z}}^{a}\right]$. This leads to

$$
\frac{\delta \chi}{\delta \tilde{A}_{\bar{z}}^{a}}=-\frac{\delta \chi}{\delta A_{\bar{z}}^{a}}=\frac{\delta \chi}{\delta E_{\bar{z}}^{a}}
$$

Then, we get

$$
E_{\bar{z}}^{a} E_{z}^{a} \psi=E_{\bar{z}}^{a} \mathcal{E}_{z}^{a} \psi
$$

which has no contribution from $\chi$. Thus, as far as only the kinetic energy term is concerned, a constant $\chi$ is sufficient [22]. If we neglect the potential energy term, the vacuum condition forces $\mathcal{E}_{z}^{a}$ to be zero. This means that the matrix $M$ has to be a holomorphic function of $\bar{z}$.

Our goal is to study the large distance behavior of the theory by neglecting second and higher order terms in $1 / m$. As we have done for TMYM theory, in subsection 4.2.1. 
neglecting the potential energy term is standard practice in these type of cases. In TMYM theory, Gauss' law forced the magnetic fields to be first order in $1 / m$ but that is not the case here. But in the literature, magnetic field contribution still gets neglected with the following reasoning. Since the energy is low, charges move very slowly and do not create significant magnetic fields [14]. When the potential term is not neglected, we expect the full solution for (5.24) to be an interpolation between the kinetic energy eigenstate and potential energy eigenstate. Since neither of these states has a first order term in $1 / m$, even if we do not neglect the potential term, there should not be any first order contribution. This result is consistent with ref. 22 . Thus, for the scales that we study, $\chi=1$ can be taken safely (at least when the potential energy is neglected), just like in TMYM theory.

\subsection{The Measure}

From (5.9), we can write the metric for the space of gauge fields $\mathfrak{A}$ as

$$
\begin{aligned}
d s_{\mathfrak{A}}^{2} & =-4 \int \operatorname{Tr}\left(\delta \tilde{A}_{\bar{z}} \delta A_{z}-\delta A_{\bar{z}} \delta \hat{A}_{z}\right) \\
& =4 \int \operatorname{Tr}\left[\tilde{D}_{\bar{z}}\left(\delta \tilde{U} \tilde{U}^{-1}\right) D_{z}\left(U^{\dagger-1} \delta U^{\dagger}\right)-D_{\bar{z}}\left(\delta U U^{-1}\right) \hat{D}_{z}\left(\hat{U}^{\dagger-1} \delta \hat{U}^{\dagger}\right)\right]
\end{aligned}
$$

Similar to the analysis in section 4.3 , the gauge invariant measure is

$$
d \mu(\mathfrak{A})=\operatorname{det}\left(\tilde{D}_{\bar{z}} D_{z}\right) \operatorname{det}\left(D_{\bar{z}} \hat{D}_{z}\right) d \mu\left(\hat{U}^{\dagger} U\right) d \mu\left(U^{\dagger} \tilde{U}\right)
$$

For a certain choice of local counter terms $\left(\int \operatorname{Tr}\left(\tilde{A}_{\bar{z}} A_{z}+\hat{A}_{z} A_{\bar{z}}\right)\right)$ we get

$$
\operatorname{det}\left(\tilde{D}_{\bar{z}} D_{z}\right) \operatorname{det}\left(D_{\bar{z}} \hat{D}_{z}\right)=\text { constant } \times e^{2 c_{A}\left(S_{W Z W}\left(U^{\dagger} \tilde{U}\right)+S_{W Z W}\left(\hat{U}^{\dagger} U\right)\right)}
$$

To simplify the notation, we will continue with defining $H_{1}=U^{\dagger} \tilde{U}$ and $H_{2}=\hat{U}^{\dagger} U$. These two matrices are $S U(N)$ gauge invariant and belong to the coset $S L(N, \mathbb{C}) / S U(N)$. Now, 
the gauge invariant measure can be written as

$$
d \mu(\mathfrak{A})=e^{2 c_{A}\left(S_{W Z W}\left(H_{1}\right)+S_{W Z W}\left(H_{2}\right)\right)} d \mu\left(H_{1}\right) d \mu\left(H_{2}\right) .
$$

\subsection{Chern-Simons Splitting}

As we have shown in section 2.12, for holomorphic polarizations, the inner product is given by

$$
\langle\psi \mid \psi\rangle=\int d \mu(\mathfrak{A}) e^{-K} \psi^{*} \psi
$$

where $K$ is the Kähler potential. Using (5.9), (5.20) and 5.21) with PW identity gives

$$
e^{-K} \psi^{*} \psi=e^{\frac{k}{2}\left(S_{W Z W}\left(H_{1}\right)-S_{W Z W}\left(H_{2}\right)\right)} \chi^{*} \chi
$$

As we have seen in subsection 5.2.1,

$$
\chi^{*} \chi=1+\mathcal{O}\left(1 / m^{2}\right)
$$

Now, using (5.33), 5.35 and (5.36), we can write the inner product as

$$
\left\langle\psi_{0} \mid \psi_{0}\right\rangle=\int d \mu\left(H_{1}\right) d \mu\left(H_{2}\right) e^{\left(2 c_{A}+\frac{k}{2}\right) S_{W Z W}\left(H_{1}\right)+\left(2 c_{A}-\frac{k}{2}\right) S_{W Z W}\left(H_{2}\right)}+\mathcal{O}\left(1 / m^{2}\right) .
$$

Here, it can be seen that the YM inner product consists of two half-level CS parts with opposite signs, that cancel as $m \rightarrow \infty$, since $H_{1}=H_{2}=H$ in this limit. The YM inner product can be written as

$$
\left\langle\psi_{0} \mid \psi_{0}\right\rangle_{Y M_{k}}=\langle\psi \mid \psi\rangle_{C S_{k / 2}}\langle\psi \mid \psi\rangle_{C S_{-k / 2}}+\mathcal{O}\left(1 / m^{2}\right)
$$

It appears that YM inner product splits into two CS terms at large distances, just as we predicted by studying its gravitational analogue 5.2 . 


\section{CHAPTER 6 \\ CONCLUSION}

We have shown that due to the existence of a mass gap, topologically massive YangMills(TMYM) theory in the near Chern-Simons(CS) limit is an almost topological field theory that consists of two copies of CS, similar to the topologically massive AdS gravity model. One copy is associated with the matrix $N$ and the other with $N^{\dagger}$, each with half the level of the original CS term in the TMYM Lagrangian. Separately momentum and position Hilbert spaces of TMYM theory can be thought of CS Hilbert spaces with half the level. In the $m \rightarrow \infty$ limit, where $N=N^{\dagger}=H$, these two CS theories add up to give one CS with the original level number $k$, as

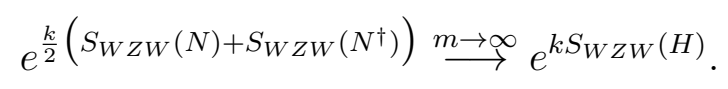

Although the integrand behaves well as $m \rightarrow \infty$, this limit is delicate for the integral measure. Studying large values of $m$ does not cause any problem, but taking it to infinity reduces the phase space dimension from four to two; thus, a change in the integral measure becomes necessary. Except for this phase space reduction, dropping the tilde symbol gives the correct CS limit in our calculations. In this limit, the metric of the space of gauge potentials reduces as

$$
-4 \int \operatorname{Tr}\left(\delta \tilde{A}_{\bar{z}} \delta A_{z}+\delta A_{\bar{z}} \delta \tilde{A}_{z}\right) \stackrel{m \rightarrow \infty}{\longrightarrow}-8 \int \operatorname{Tr}\left(\delta A_{\bar{z}} \delta A_{z}\right)
$$

For the left hand side of 6.2 , the measure is given by 4.37) while for the right hand side, it is given by 3.22 . Thus, the measure needs to be replaced with 3.22 in the pure CS limit. Although this reduction occurs beautifully in the metric, the volume (4.37) does not 
automatically reduce to $(3.22)$ in our notation. Not switching to the correct volume element results in duplicate integration over $H$, since in the pure CS limit $N=N^{\dagger}=H$. This comes from the fact that the phase space of TMYM theory consists of two CS phase spaces.

A different problem exists for the pure Yang-Mills(YM) limit $m \rightarrow 0$. In this case, dimensionality of the phase space does not change, but since $E$-fields do not gauge transform like $\tilde{A}$ fields, our parametrization and measure do not work. But the main problem with studying the pure YM limit comes from not knowing the magnetic field contribution in the wave-functional, which becomes the dominant part in this limit. To get the magnetic field contribution, (5.24) needs to be fully solved without using the strong coupling limit.

CS splitting does not seem to appear if one uses the real polarization that makes $\psi=\psi\left[A_{z}, A_{\bar{z}}\right]$. This can be seen clearly in refs. $13-15$. These earlier works study TMYM theory in a perspective where YM theory is perturbed by a CS term, while our work is exactly the opposite. Ideally, all polarizations should lead to the same inner product; but in this case, different polarizations create different mathematical difficulties that force one to focus on certain scales. For TMYM theory, holomorphic polarization facilitates studies near the CS limit, while the real polarization is suitable for the near YM limit [51]. The difficulties that arise when using the real polarization can be summarized as follows. First, $A_{z}$ and $A_{\bar{z}}$ do not commute in the pure CS limit; the wave-functional cannot depend on both, at very large distances. This makes it impossible to check the CS limit of the wave-functional. But in the holomorphic polarization TMYM wave-functional reduces to CS wave-functional nicely. Second, in the real polarization, the wave functional cannot depend on $E$-fields. But in the near CS limit, $E$-fields dominate the Hamiltonian while $B$-fields are negligible. This 
creates a difficulty in studying large distances. Since CS splitting occurs at a scale where the first order $E$-field contributions are important, it is not surprising to not see this feature in a polarization that cannot resolve this scale. Similarly, holomorphic polarization is not helpful in studying smaller scales where $B$-field contributions are important, since $\psi\left[A_{\bar{z}}, \tilde{A}_{\bar{z}}\right]$ does not depend on $B$-fields. Thus, to study the near CS limit, holomorphic polarization should be chosen. Therefore, our results cannot be compared [51] with the results of refs. $13-15$, since they focus on a different scale where CS splitting does not occur.

Usually in the literature, the constant $k / 4 \pi$ is not inserted in the YM term and this leads to a mass gap $\propto k m$. Thus, a question may arise on whether or not taking $k$ to be a large integer causes any problem. In our calculations, it did not cause any problem in obtaining a topological theory at large distances. This indicates that having a large level number does not alter the existence of the mass gap in TMYM theory, although we do not provide a proof.

In section 4.4 , by writing 4.52 we showed that loop operator expectation values of CS and TMYM theories are related at large distances. This equivalence tells us that expectation values of both Wilson loops and 't Hooft loops in TMYM theory are equal to CS Wilson loop expectation values, up to a change in level number. A more interesting result is that the expectation value of the product of these loops in TMYM theory is equal to the product of Wilson loop expectation values in CS theory. These results show that not only in the pure CS limit but also in the near CS limit, the observables of TMYM theory are link invariants. Both Wilson loops and 't Hooft loops can separately form links that satisfy the skein relation $(3.30)$, but a mixed link of these loops does not, even though it is still a 
link invariant.

In chapter 5, we have shown that YM theory acts like a topological theory at certain limited scales, just like TMYM theory. When the distance is large enough but finite, YM theory splits into two CS terms with levels $k / 2$ and $-k / 2$ very similar to the splitting of topologically massive AdS gravity model. At very large distances, these two CS terms cancel to make YM theory trivial. Together with our calculation for TMYM theory, we have shown that both YM and TMYM theories exhibit CS splitting at large scales, just as predicted by their gravitational analogues. The methods that we have introduced in section 4.4 can be used to exploit this limited topological region to incorporate link invariants for pure YM theory as well. 


\section{REFERENCES}

[1] E. Witten. Quantum Field Theory and the Jones Polynomial. Communications in Mathematical Physics, 121:351, 1989.

[2] P. Cotta-Ramusino, E. Guadagnini, M. Martellini, and M. Mintchev. Quantum Field Theory and Link Invariants. Nuclear Physics B, B330:557, 1990.

[3] J.F. Schonfeld. A Mass Term for Three-Dimensional Gauge Fields. Nuclear Physics B, 185:157-171, 1981.

[4] S. Deser, R. Jackiw, and S. Templeton. Topologically Massive Gauge Theories. Annals of Physics, 140:372 - 411, 1982.

[5] S. Deser, R. Jackiw, and S. Templeton. Three-Dimensional Massive Gauge Theories. Physical Review Letters, 48:975-978, 1982.

[6] S. Deser and R. Jackiw. "Self-Duality" of Topologically Massive Gauge Theories. Physics Letters B, 139:371 - 373, 1984.

[7] D. Gonzales and A.N Redlich. A Gauge Invariant Action for $(2+1)$-Dimensional Topologically Massive Yang-Mills theory. Annals of Physics, 169:104 - 116, 1986.

[8] P.A. Horvathy and C. Nash. Geometric View On Topologically Massive Gauge Theories. Physical Review D, 33(6):1822, 1986.

[9] D. Evens and G. Kunstatter. Hamiltonian Analysis of Topologically Massive Yang-Mills Theory. Physical Review D, 37:435-440, 1988.

[10] J.M. Martinez-Fernandez and C. Wotzasek. Constrained Quantization of a Topologically Massive Gauge Theory in (2+1)-Dimensions. Zeitschrift für Physik C Particles and Fields, 43:305-312, 1989.

[11] G. Giavarini, C.P. Martin, and F. Ruiz Ruiz. Chern-Simons Theory as the Large-Mass Limit of Topologically Massive Yang-Mills Theory. Nuclear Physics B, 381:222 - 280, 1992.

[12] G. Giavarini, C.P. Martin, and F. Ruiz Ruiz. Abelian Chern-Simons Theory as the Strong Large Mass Limit of Topologically Massive Abelian Gauge Theory: The Wilson loop. Nuclear Physics B, B412:731-750, 1994. 
[13] M. Asorey, S. Carlip, and F. Falceto. Chern-Simons States and Topologically Massive Gauge Theories. Physics Letters B, 312:477-485, 1993.

[14] G. Grignani, G. Semenoff, P. Sodano, and O. Tirkkonen. G/G Models as the Strong Coupling Limit of Topologically Massive Gauge Theory. Nuclear Physics B, 489:360 384, 1997.

[15] D. Karabali, C.-J. Kim, and V.P. Nair. Gauge Invariant Variables and the Yang-MillsChern-Simons Theory. Nuclear Physics B, B566:331-347, 2000.

[16] G. Dunne, A. Kovner, and B. Tekin. Magnetic Symmetries and Vortices In ChernSimons Theories. Physical Review D, 63:025009, 2000.

[17] F. Canfora, A. J. Gómez, S. P. Sorella, and D. Vercauteren. Study of Yang-Mills-ChernSimons Theory In Presence of the Gribov Horizon. Annals of Physics, 345:166 - 177, 2014.

[18] V.E.R. Lemes, C. Linhares de Jesus, C.A.G. Sasaki, S.P. Sorella, and L.C.Q.Vilar. A Simple Remark on Three-Dimensional Gauge Theories. Physics Letters B, B418:324328, 1998.

[19] V.E.R. Lemes, C. Linhares de Jesus, S.P. Sorella, L.C.Q. Vilar, and O.S. Ventura. Chern-Simons as a Geometrical Setup for Three-Dimensional Gauge Theories. Physical Review D, D58:045010, 1998.

[20] D. Karabali and V.P. Nair. Gauge Invariance and Mass Gap in (2+1)-Dimensional Yang-Mills Theory. International Journal of Modern Physics A, A12:1161-1172, 1997.

[21] R.P. Feynman. The Qualitative Behavior of Yang-Mills Theory In 2+1 Dimensions. Nuclear Physics B, 188(3):479-512, 1981.

[22] D. Karabali, C.-J. Kim, and V.P. Nair. On the Vacuum Wave Function and String Tension of Yang-Mills Theories In (2+1)-Dimensions. Physics Letters B, B434:103-109, 1998.

[23] M. J. Teper. SU (N) gauge theories in 2+1 dimensions. Physical Review D, 59(1):014512, 1998.

[24] B. Lucini and M. Teper. SU (N) Gauge Theories In 2+1 Dimensions: Further Results. Physical Review D, 66(9):097502, 2002.

[25] B. Bringoltz and M. Teper. A Precise Calculation of the Fundamental String Tension In SU (N) Gauge Theories In 2+1 Dimensions. Physics Letters B, 645(4):383-388, 2007. 
[26] S.-S. Chern and J. Simons. Characteristic forms and geometric invariants. Annals of Mathematics, pages 48-69, 1974.

[27] R Jackiw and S Templeton. How Super-Renormalizable Interactions Cure Their Infrared Divergences. Physical Review D, 23(10):2291, 1981.

[28] M. Nakahara. Geometry, Topology and Physics. CRC Press, 2003.

[29] A. Quadri. Comments on the Equivalence Between Chern-Simons Theory and Topological Massive Yang-Mills Theory in 3-D. Journal of High Energy Physics, 0211:030, 2002.

[30] E. Witten. 2+1 Dimensional Gravity as an Exactly Soluble System. Nuclear Physics $B, 311(1): 46-78,1988$.

[31] S. Carlip, S. Deser, A. Waldron, and D.K. Wise. Topologically Massive AdS Gravity. Physics Letters B, 666(3):272 - 276, 2008.

[32] S. Carlip, S. Deser, A. Waldron, and D.K. Wise. Cosmological Topologically Massive Gravitons and Photons. Classical and Quantum Gravity, 26(7):075008, 2009.

[33] V.P. Nair. Quantum Field Theory: A modern Perspective. Springer, 2005.

[34] E. Witten. Non-Abelian Bosonization In Two Dimensions. Communications in Mathematical Physics, 92:455-472, 1984.

[35] A. Polyakov and P.B. Wiegmann. Theory of Nonabelian Goldstone Bosons In Two Dimensions. Physics Letters B, 131:121 - 126, 1983.

[36] A.M. Polyakov and P.B. Wiegmann. Goldstone Fields In Two Dimensions With Multivalued actions. Physics Letters B, 141:223 - 228, 1984.

[37] D. Karabali and V.P. Nair. A Gauge-Invariant Hamiltonian Analysis for Non-Abelian Gauge Theories in (2+1) Dimensions. Nuclear Physics B, 464:135 - 152, 1996.

[38] M. Bos and V.P. Nair. Coherent State Quantization of Chern-Simons Theory. International Journal of Modern Physics A, A5:959, 1990.

[39] P. Ramond. Field Theory: A Modern Primer. Westview Press, 1997.

[40] W. Greiner, D.A. Bromley, S. Schramm, and E. Stein. Quantum Chromodynamics. Springer, 2007.

[41] G. Ripka. Dual Superconductor Models of Color Confinement, volume 639. Springer, 2004. 
[42] M.F. Atiyah. The Geometry and Physics of Knots. Cambridge University Press, 1990.

[43] J.W. Alexander. Topological Invariants of Knots and Links. Transactions of the American Mathematical Society, 30(2):275-306, 1928.

[44] V.F.R. Jones. A Polynomial Invariant For Knots Via Von Neumann Algebras. Bulletin of the American Mathematical Society, 12(1):103-111, 1985.

[45] M.F. Atiyah. New Invariants of Three and Four Dimensional Manifolds. The Mathematical Heritage of Hermann Weyl. Proc. Symp. Pure Math., 48:285-299, 1988.

[46] P. Freyd, D. Yetter, J. Hoste, W.B.R. Lickorish, K. Millett, and A. Ocneanu. A New Polynomial Invariant of Knots and Links. Bulletin of the American Mathematical Society, 12(2):239-246, 1985.

[47] B. C. Hall. Quantum Theory for Mathematicians. Springer, 2013.

[48] S. Axelrod, S.D. Pietra, and E. Witten. Geometric Quantization of Chern-Simons Gauge Theory. Journal of Differential Geometry, 33:787-902, 1991.

[49] T. Yildirim. Topologically Massive Yang-Mills Theory and Link Invariants. arXiv preprint arXiv:1311.1853, 2013.

[50] T. Yildirim. Chern-Simons Splitting of 2+1D Pure Yang-Mills Theory at Large Distances. arXiv preprint arXiv:1410.8593, 2014.

[51] V.P. Nair. Private communication. 